\title{
Ultrasonic characterization of the anisotropic behavior of air-saturated porous materials
}

\author{
Bernard Castagnede *, Achour Aknine, Manuel Melon, Claude Depollier \\ LAUM/IAM (UMR CNRS 6613), 72085 Le Mans, Ceder 9. France'
}

\begin{abstract}
This paper provides a comprehensive review of the propagation of ultrasonic waves in anisotropic porous materials. The equivalent fluid model (or Allard-Johnson theory) which is relevant for air-saturated porous media is described. It takes into account viscous and thermal losses occurring during the movement of the fluid within the motionless solid frame. When the skeleton is moving as well, the coupled Biot theory should instead be used. This theory becomes intricate when anisotropy is considered due to a very large number of physical parameters to be determined. A strong formal correspondence between the anisotropic Biot wave and the thermal wave of dynamic thermoelasticity in non-porous media is outlined. Standard ultrasonic methods, generally used at low frequency (i.e. $20-500 \mathrm{kHz}$ ) are very effective in order to characterize anisotropy in porous media. Both reflection and transmission configurations have been used. Special attention has been devoted to the measurements of the anisotropic tortuosity, but also to the viscous and thermal characteristics lengths. Finally, some inverse problems related to these measurements are solved and others, which are still open, are presented. O 1998 Elsevier Science B.V.
\end{abstract}

Keywords: Anisotropy; Allard theory; Biot theory; Thermal wave; Reflection coefficient; Transmission: Anisotropic tortuosity; Characteristic lengths

\section{Introduction}

Porous media filled with air, such as plastic foams, fibrous mats and various felts, are heavily used in the automotive, aeronautical and building industries to damp and attenuate sound waves. During the last 5 years, low frequency (i.e. in the range $20-500 \mathrm{kHz}$ ) ultrasonic techniques have amply proven to be an invaluable tool to probe and decipher the intricacies of the acoustical properties of these materials.

The present review starts by briefly exposing some of the present theoretical models. One distinguishes the two basic cases where the solid frame is moving or not. The first case refers to the Biot theory $[1,2]$, where the coupling is responsible for the existence of an additional slow longitudinal wave. It can be easily observed with porous structures saturated by an heavy fluid (such as water). For air-saturated porous materials, the Biot theory is often required to describe the acoustics of layered materials including porous, elastic and viscoelastic media. We shall restrict ourself in this review to

\footnotetext{
* Corresponding author. Fax: + 33243833520 ;

e-mail: casta(c)asterix.univ-lemans. fr
}

homogeneous porous single layer, because the acoustics of layered media including porous materials is well known, as seen from the available literature. For the simple case of a motionless skeleton (i.e. for a single porous layer), one can use the Johnson-Allard theory [3] which concentrates on the porous material seen as an 'equivalent fluid' $[4,5]$.

Often, porous media are anisotropic and this feature adds complexity in terms of the description, increasing the number of independent physical parameters that are required in the model. For instance, for a thin layer of porous medium of hexagonal symmetry (i.e. being transversely isotropic), the Biot description necessitates 11 parameters (five elastic constants, two Biot coupling coefficients, the compressibility of the fluid, and three densities for the fluid, the frame and the coupling term).

Various numerical and experimental results are also presented. Emphasis is given to the anisotropic version of the Johnson-Allard model because it is the case which is most commonly encountered with air-saturated porous materials. For such a case one needs to determine five parameters: the porosity $\phi$, the flow resistivity $\eta / k_{0}$, the tortuosity $\alpha$, the viscous and the thermal characteristic lengths $A, A^{\prime}$ (see the next section for further explana- 
tion; nomenclature is given at the end of the paper). For a material having hexagonal symmetry there are instead eight parameters. Reflection and transmission coefficients are studied in some details. A section is devoted to the theory of dynamic thermoelasticity because of the strong analogy between such model and the Biot theory (as suggested 40 years ago by M.A. Biot himself). The fit between theoretical predictions and the available experimental data is generally excellent, enabling in turn one to solve various inverse problems (e.g. to accurately determine some of the above parameters).

\section{Theory of the equivalent fluid}

In this model, one begins by dealing with the two fundamental equations of linear acoustics in an isotropic porous medium, which, respectively, are the Euler equation and the equation of mass conservation associated with the behavior (or adiabatic) equations, as written below:

$\rho_{\mathrm{f}} \alpha(\omega) \frac{\partial v_{i}}{\partial t}=-\nabla_{i} p$

$\frac{1}{K_{\mathrm{a}}} \beta(\omega) \frac{\partial p}{\partial t}=-\nabla \cdot v$.

In these equations $p$ and $v$ are the acoustic pressure and the acoustic particle displacement derivative (by the term 'acoustic' we denote the small difference of these quantities, around the values of the parameters at rest, which are due to the acoustic wave). The wavespeed in the porous material is then derived from these two equations by elementary manipulations and yields the usual standard equation:

$c(\omega)=\sqrt{\frac{K_{\mathrm{a}}}{\rho_{\mathrm{f}} \alpha(\omega) \beta(\omega)}}$,

with $K_{\mathrm{a}}=\gamma P_{0}$, and where $\alpha(\omega)$ and $\beta(\omega)$ are, respectively, the dynamic tortuosity and the relative dynamic compressibility of the fluid included in the porous media. These two response factors are complex functions which heavily depend on the frequency. They take into account the inertial interaction between the fluid and the skeleton of the porous material and the viscous and thermal losses which are localized at high frequencies at the boundary between the two domains. The theoretical expressions for such quantities are given in Refs. $[5,6]$ :

$$
\begin{aligned}
& \alpha(\omega)=\alpha_{\infty}\left(1+\frac{1}{\mathrm{j} X} \sqrt{1+\frac{M}{2} \mathrm{j} X}\right), \\
& \beta(\omega)=\gamma-\frac{\gamma-1}{\left(1+\frac{1}{\mathrm{j} X^{\prime}} \sqrt{1+\frac{M^{\prime}}{2} \mathrm{j} X^{\prime}}\right)},
\end{aligned}
$$

where

$X=\frac{\omega \alpha_{\infty} \rho_{\mathrm{f}} k_{0}}{\eta \phi}, \quad M=\frac{8 \alpha_{\infty} k_{0}}{\phi A^{2}}$,

and

$X^{\prime}=\frac{\omega \rho_{\mathrm{r}} k_{\mathrm{o}}}{\eta \operatorname{Pr} \phi}, \quad M^{\prime}=\frac{8 k_{0}^{\prime}}{\phi \Lambda^{\prime 2}}$

In these expressions $\mathrm{j}=\sqrt{-1}, \omega=2 \pi f$ represents the angular frequency, $\gamma$ the ratio of specific heat (or adiabatic constant), $\eta$ the fluid viscosity, $\operatorname{Pr}$ the Prandtl number, $\rho_{\mathrm{f}}$ the density of air at rest, $\phi$ the porosity, $k_{0}$ the static permeability, $k_{0}^{\prime}$ the thermal permeability, $\alpha_{\infty}$ the tortuosity, $A$ and $A^{\prime}$ the viscous and thermal characteristic lengths [7-9]. Eq. (4), in a slightly different form due to the \pm sign convention in the $\exp (\mathrm{j} \omega t)$ term, was originally introduced in 1987 by Johnson in a seminal paper [10] which described the damping of acoustical waves in a porous material caused by viscous effects. Subsequently, other authors did study with similar tools the acoustics of porous media (e.g. Refs. $[11,12]$ ).

Such a model was then extended in 1992 by Allard and Champoux [13] to include the thermal effects [Eq. (5)]. The parameter $k_{0}^{\prime}$ in Eq. (7) was introduced by Lafarge [5] to describe the additional attenuation of the acoustical waves due to thermal exchanges taking place at the surface of the pores. This last parameter cannot be easily measured by a nonacoustical technique. In practice, the $M^{\prime}$ parameter is chosen to be equal to one which is the exact value for a material with parallel cylindrical pores. This approximation is valid for a large number of porous acoustical materials. When dealing with ultrasound where the viscous skin depth $\delta$ is small compared to the characteristic lengths, the $\alpha(\omega)$ and $\beta(\omega)$ functions are developed as:

$\alpha(\omega)=\alpha_{\infty}\left(1+(1-j) \frac{\delta}{A}\right)$,
$\beta(\omega)=\gamma-(\gamma-1)\left[1-(1-j) \frac{\delta}{B \Lambda^{\prime}}\right]$,

with

$\delta=\sqrt{\frac{2 \eta}{\rho_{\mathrm{f}} \omega}}$

and $B=\sqrt{\mathrm{Pr}}$, which provide the wavespeed at high frequencies:

$c(\omega)=\frac{c_{0}}{\sqrt{\alpha_{\infty}}}\left[\begin{array}{ll}1 & \delta \\ & 2\end{array}\left(\frac{1}{A}+\frac{\gamma-1}{B \Lambda^{\prime}}\right)\right]$ 
with

$c_{0}=\sqrt{\frac{K_{\mathrm{a}}}{\rho_{\mathrm{f}}}}$

which is the sound velocity in air at rest.

The validity domain of the high frequency approximation is visualized in Fig. 1, taken from Ref. [14], for the real part of the relative dynamic compressibility. One can observe that above $10-15 \mathrm{kHz}$ the asymptotic expressions of the relative dynamic compressibility are sufficiently precise. Moreover, a comparison at low frequencies (in the audio range) between the complete model (with no high frequencies approximation) is shown for a fibrous material on Fig. 2 laken from Ref. [15].

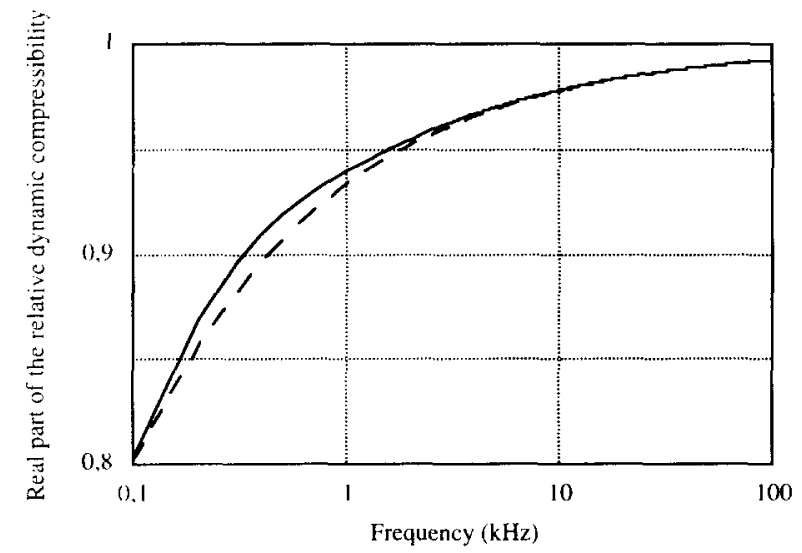

Fig. 1. Real part of the dymanic compressibility. (_-) No approximation; (- - ) high frequency approximation. The parameters used in the calculations are: $x_{\infty}=1.2 ; \phi=0.98 ; \Lambda=1.2 \times 10^{-4} \mathrm{~m}$, $\Lambda^{\prime}=5.0 \times 10^{-4} \mathrm{~m}$.

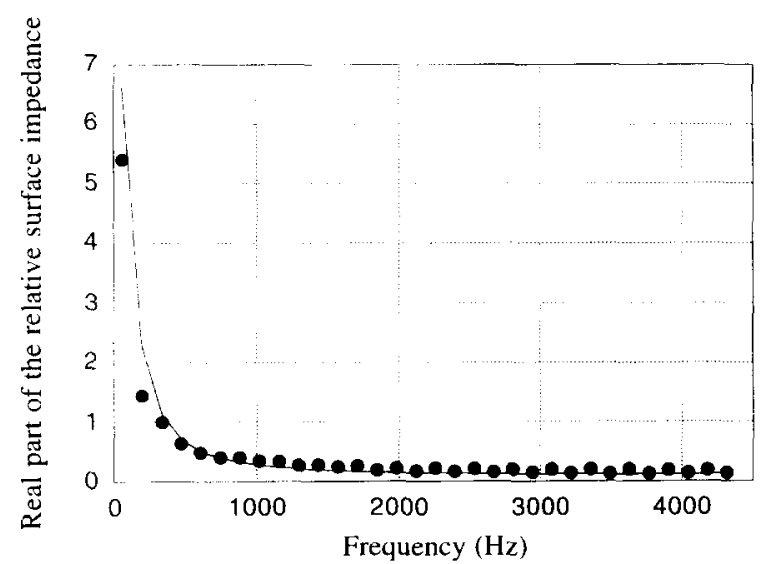

Fig. 2. Real part of the surface impedance for a fibrous material as determined with the TMTC method. The physical parameters used in the numerical simulation are the following: $\left.\phi=0.99 ; \quad \eta / k_{0}\right)=$ $6442 \mathrm{~N} \mathrm{~m}^{-4} \mathrm{~s}^{-1} ; x_{\infty}=1.00 ; \Lambda=1.66 \times 10^{-4} \mathrm{~m} ; \Lambda=4.98 \times 10^{-4} \mathrm{~m}$. The solid line represents the numcrical results, and the dots the experimental data.

\section{Anisotropic parameters of the model}

When anisotropy is present, the above model should be modified accordingly. A major difference stems from the response factor $\alpha(\omega)$ which becomes a second rank tensor as stated by the modified Euler equation:

$\rho_{\mathrm{r}} \tilde{\boldsymbol{\alpha}}_{i j}(\omega) \frac{\hat{C} v_{j}}{\partial t}=-\nabla_{i} p$

whereas Eq. (2) is unaffected. Neglecting viscous losses, $\tilde{\boldsymbol{\alpha}}_{i j}(\omega)$ is a real symmetric tensor. This important symmetry property can be shown by using energy considerations completely analogous to those used in electrodynamics for demonstrating the symmetrical character of the dielectric tensor of a medium. Thus, the tortuosity tensor diagonalizes in a system of three orthogonal axes - the so-called principal axes. In the general case where viscous losses are present, the symmetry of the complex tensor $\tilde{\alpha}_{i j}(\omega)$ is maintained and can be viewed as an example of the general Onsager's reciprocity rules. However, the real and imaginary part of the tensor $\tilde{x}_{i j}(\omega)$ need not diagonalize in the same system of principal axes. Moreover, the principal axes themselves may be frequency dependent. Such dispersion of the principal axes does not occur a priori for transversally isotropic materials such as the ones studied in this paper. In addition, ultrasonic measurements lie in the highfrequency regime where the imaginary part of the tensors $\tilde{\boldsymbol{\alpha}}_{i j}(\omega)$ is small compared to the real part. The shape of this tensor is in the general case an ellipsoid, which is quite analogous to the index of refraction tensor in the optics of crystals. The intersections of this sheet along the principal acoustic axes of the porous solid provide the three principal values of the tensor. Because we restrict ourself here to hexagonal anisotropy, there is a plane which should be isotropic. It is noted as plane $p$, and the normal plane to it by $n$. The tortuosity tensor assumes the following form:

$\tilde{\alpha}_{i j}(\omega)=\left(\begin{array}{ccc}\alpha_{\mathrm{p}}(\omega) & 0 & 0 \\ 0 & \alpha_{\mathrm{p}}(\omega) & 0 \\ 0 & & \alpha_{\mathrm{n}}(\omega)\end{array}\right)$

With the same notation on hand for the two principal acoustical axes ( $\mathrm{p}$ for in plane, and $\mathrm{n}$ for normal to the porous layer), the $\alpha(\omega)$ response function is then defined by the following expression:

$\alpha_{k}(\omega)=x_{\infty}\left(1+\frac{1}{\mathrm{j} X_{k}} \sqrt{1+\frac{M_{k}}{2} \mathrm{j} X_{k}}\right), \quad k=\mathrm{n}, \mathrm{p}$,

where the $X_{k}$ and $M_{k}$ parameters are given by

$X_{k}=\frac{\omega \alpha_{\infty \mathrm{k}} \rho_{\mathrm{f}} k_{0 k}}{\eta \phi} ; \quad M_{k}=\frac{8 k_{0 k} \alpha_{\circ 0 k}}{\phi A_{k}^{2}}$. 
The $\beta(\omega)$ response function is unchanged, as given by Eq. (5).

The wavevector in the transversely isotropic case, is expressed by the two components:

$k_{\mathrm{m}}=\left(\begin{array}{l}k_{x} \\ k_{z}\end{array}\right)=\left(\begin{array}{c}\frac{\omega}{c_{0}} \sin \theta \\ k_{\mathrm{n}} \sqrt{1-\frac{k_{x}^{2}}{k_{\mathrm{p}}^{2}}}\end{array}\right)$,

with

$k_{k}=\omega \sqrt{\frac{\rho_{\mathrm{f}} \alpha_{k}(\omega) \beta(\omega)}{K_{\mathrm{a}}}}, \quad k=\mathrm{n}, \mathrm{p}$,

where $k_{x}$ and $k_{z}$ denotes the components of the wavevector in the fluid saturating the structure (the propagation lying in plane $x z$, where $z$ stands for the normal $n$ and $x$ for the in-plane orthogonal axis $p$ ). In terms of the physical parameters of the porous materials, beside the tortuosity which becomes anisotropic, the porosity and the thermal characteristic length remain scalars and thus are still isotropic. On the other hand, the flow resistivity $\eta / k_{0}$, and the viscous characteristic length become anisotropic as well, and this point is discussed in the next section.

\section{Measurements of the coefficient of reflection}

When dealing with short ultrasonic signals (typically lasting a few microseconds), the reflection coefficient at the boundary of an isotropic porous slab is then given by [14]

$$
R_{\mathrm{i}}=\frac{\frac{\cos \theta}{Z_{\mathrm{f}}}-\frac{k_{z} \phi}{k_{\mathrm{n}} Z_{\mathrm{m}}}}{\frac{\cos \theta}{Z_{\mathrm{f}}}+\frac{k_{z} \phi}{k_{\mathrm{n}} Z_{\mathrm{m}}}}
$$

where $Z_{\mathrm{f}}$ and $Z_{\mathrm{m}}$, which are respectively the characteristic impedances of the fluid outside and inside the porous media, take the following forms:

$Z_{\mathrm{f}}=\rho_{\mathrm{f}} c_{0}, \quad Z_{\mathrm{m}}=\sqrt{\frac{\rho_{\mathrm{f}} K_{\mathrm{a}} \alpha(\omega)}{\beta(\omega)}}$.

In this case, the interferences between the multiple reflections in the porous material does not exist when the duration of the signal is lower than the quantity $[2 L / c(\omega)]$. It should be noted that in the audible frequency range, such interferences must be taken into account. One must use a theoretical reflection coefficient calculated from the combination of the transfer matrix of a porous media immersed in air with the appropriate boundary conditions [6]. For a transversally isotropic

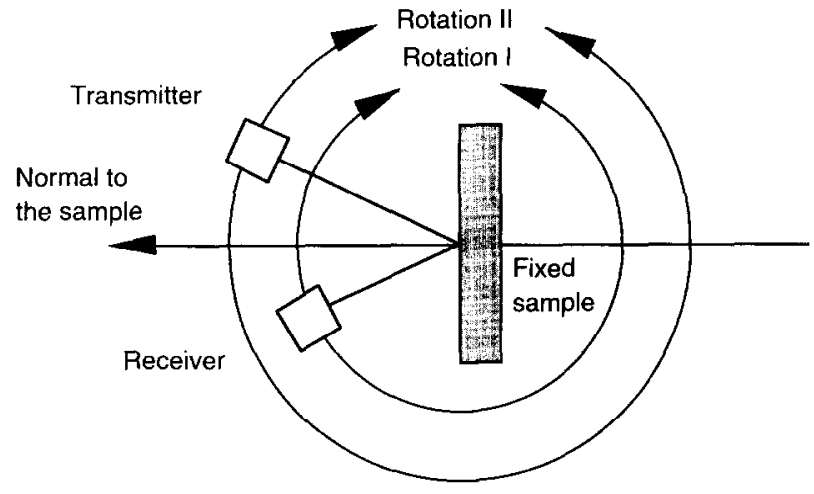

Fig. 3. Schematic diagram of the experimental set-up in the reflection configuration.

material, the reflection coefficient is given by Eq. (17) with $Z_{\mathrm{m}}=Z_{\mathrm{n}}$ and $\alpha(\omega)=\alpha_{\mathrm{n}}(\omega)$, where $Z_{\mathrm{n}}$ is the characteristic impedance of the saturating fluid along the normal direction.

A critical angle $\theta_{0}$ where the reflection coefficient is equal to 0 is calculated when frequency tends to infinity:

$\sin \theta_{0}=\sqrt{\frac{\alpha_{\infty \mathrm{n}}-\phi^{2}}{\alpha_{\infty \mathrm{n}}-\frac{\phi^{2}}{\alpha_{\infty \mathrm{p}}}}}$.

Reflection coefficient measurements in air are greatly facilitated by using ultrasonic techniques, as is reported in this work (also see Ref. [16]). The reflection coefficient measurements versus the incidence angle have been performed with an ultrasonic reflectometer designed at LAUM (Fig. 3). Because of the very weak signals obtained after reflection, a 5058PR pulser-receiver from Panametrics which provides $900 \mathrm{~V}$ peak-to-peak and a $90 \mathrm{~dB}$ dynamic range has been used. The signals captured by a 9310 LeCroy digitizing oscilloscope were processed by a Macintosh Quadra 950 computer running LabVIEW 3.0. The amplitude measurements were performed in the Fourier domain by using the amplitude spectrum. A precision of the order of $1 \%$ was achieved. The comparison between theory and experiments (a characteristic example is shown in Fig. 4) shows that when the material is assumed to be isotropic the agreement is poor. When anisotropy is taken into account, which should definitely be done for the Tramico material as can be seen (in the caption of Fig. 4 from the values of the tortuosity along the principal directions), the agreement becomes fair. Other materials tested in the same way exhibit a similar trend in the results.

\section{Transmission coefficient when the frame is motionless}

The coefficient of transmission of an isotropic porous slab of thickness $L$ when the frame is motionless is 


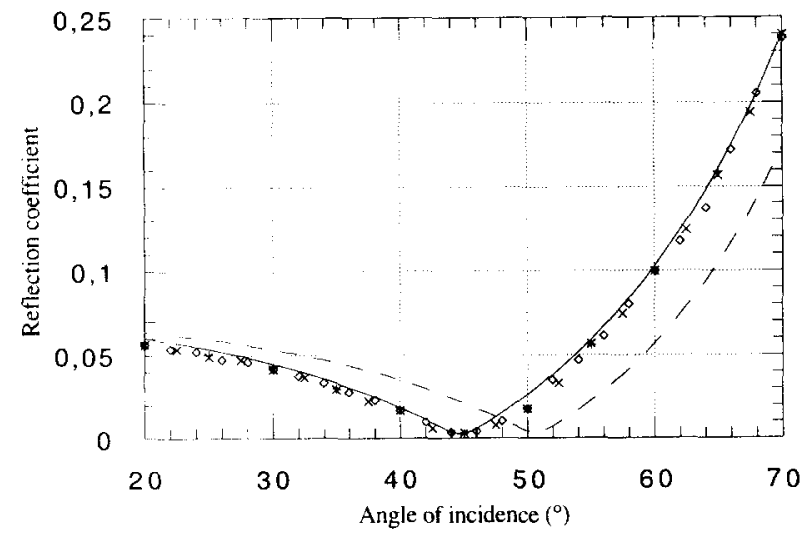

Fig. 4. Reflection coefficient versus incidence angle. Comparison between theoretical and experimental values. The theoretical values are computed with the following parameters: $(1)$ when the material is assumed to be isotropic (dashed line) $\left(\eta / k_{0}\right)=5000 \mathrm{~N} \mathrm{~m}^{-4} \mathrm{~s}^{-1} ; \phi=$ 0.98; $A=1.2 \times 10^{-4} \mathrm{~m} ; A^{\prime}=5 \times 10^{-4} \mathrm{~m} ; x_{\infty}=1.2$; and (2) when it is assumed to be anisotropic (continuous line) $\left(\eta / k_{0 \mathrm{n}}\right)=4900 \mathrm{~N} \mathrm{~m}^{* 4}$ $\mathrm{s}^{-1} ;\left(\eta / k_{\mathrm{op}}\right)=6900 \mathrm{~N} \mathrm{~m}^{-4} \mathrm{~s}^{-1}: \quad \phi=0.98 ; \quad A_{n}=2.2 \times 10^{-4} \mathrm{~m} ; A_{\mathrm{p}}=2.0$ $\times 10^{-4} \mathrm{~m} ; A^{\prime}=5 \times 10^{-4} \mathrm{~m} ; x_{x \mathrm{n}}=1.2 ; \alpha_{\infty \mathrm{p}}=1.4$.

defined, in the high frequency assumption which is relevant with ultrasound, by a very simple relationship (e.g. see Refs. [14,17]):

$$
T_{i}=\frac{\frac{4 k_{z} \phi \cos \theta}{Z_{f} Z_{\mathrm{m}} k_{\mathrm{n}}}}{\left(\frac{\cos \theta}{Z_{f}}+\frac{k_{z} \phi}{k_{\mathrm{n}} Z_{\mathrm{m}}}\right)^{2}} \mathrm{e}^{-\mathrm{j} k_{z} L_{.}},
$$

with

$Z_{\mathrm{m}}=\rho_{\mathrm{f}} c_{0} \sqrt{\alpha_{\infty}}\left[1+\frac{\delta(1-\mathrm{j})}{2}\left(\frac{1}{A}-\frac{\gamma-1}{B A^{\prime}}\right)\right]$,

and where $Z_{\mathrm{f}}=\rho_{\mathrm{f}} \mathrm{c}_{0}$ represents the characteristic impedance of air.

In most cases, for absorbing porous materials, the porosity as well as the tortuosity are close to 1 . It is then evident to note that $\left(\sqrt{\alpha_{\infty}} / \phi\right)+\left(\phi / \sqrt{\alpha_{\infty}}\right) \simeq 2$, when the porosity is between 0.9 and 1 , and the tortuosity smaller than 1.4. Accordingly, with the help of that approximation, there exists a simplier form of the transmission coefficient as given by

$\lim _{j \rightarrow \infty} T_{\mathrm{i}}=\exp \left(-\frac{\omega}{\mathcal{C}_{0}} \sqrt{\alpha_{\infty}}\left[\frac{\delta}{2}\left(\frac{1}{A}+\frac{\gamma-1}{B A^{\prime}}\right)\right] L\right)$.

This last expression indicates that the transmission cocfficient is directly linked to the tortuosity as well as to the characteristic lengths. A complete discussion on this question can be found in Ref. [18]. Furthermore, a detailled analysis of the two-dimensional scan images (see Fig. 5 for a sketch of the configuration and refer to Refs. $[14,18]$ for such experimental two-dimensional scans) unambiguously demonstrate that there exists a strong correlation between the spatial variations of the tortuosity and those of the transmission coefficient; this result in turn is directly predicted from Eq. (22). From that same equation, it is then possible to numerically recover the two-dimensional scan image of the transmission from those of the experimental tortuosity. This comparison is not really satisfactory. Some losses are responsible for the inadequate comparison, because the experimental transmission coefficient is systematically below the theoretical predictions made from Eq. (22) with the appropriate material parameters (here the tortuosity and the characteristic lengths). An example is provided in Fig. 6 showing that the recovered viscous characteristic length is $40 \%$ smaller than its actual value as determined by other independent methods. When anisotropy is present the transmission coefficient at normal incidence is simply provided by Eq. (20) where $k_{\mathrm{m}}=k_{\mathrm{n}}$ and $Z_{\mathrm{m}}=Z_{\mathrm{n}}$ (see Ref. [14], p. 99 for further details).

\section{Anisotropy of the tortuosity}

Some measurements of the dynamic tortuosity have been performed with narrowband piezoelectric transducers on reticulated plastic foams. Some preliminary results have been reported [19]. Further experimental results related to the anisotropic nature of the material have been obtained by probing the tortuosity versus transmission angle [20]. A simple numerical routine has been implemented in order to recover the tortuosity along principal directions. These predicted values compare well with measurements done directly along the principal acoustic axes of the samples. Slight angular deviations of the principal axes themselves have also been observed.

Air-saturated materials are, to some extent, anisotropic. An indirect way to characterize such anisotropy was recently achieved by measuring the coefficient of reflection versus incidence angle for reticulated plastic foams (as discussed in Section 4). The comparison between the experimental data and the results drawn from the Johnson-Allard theory is adequate only when some anisotropy of the relevant parameters is taken into account. Furthermore, the predicted anisotropic parameters were in good agreement with the measured values.

The amount of anisotropy that is observed in reticulated plastic foams is slight, in the order of $10-20 \%$. In fact, these materials are also heterogeneous, and the acoustical properties depend on the location which is probed. The observed variations of the phase wavespeed and transmission coefficient at normal incidence are again in the range of $10-20 \%$. The next idea is to introduce experimental routines which may decipher the influence of anisotropy and heterogeneity which are of the same order of magnitude. One can define the real 


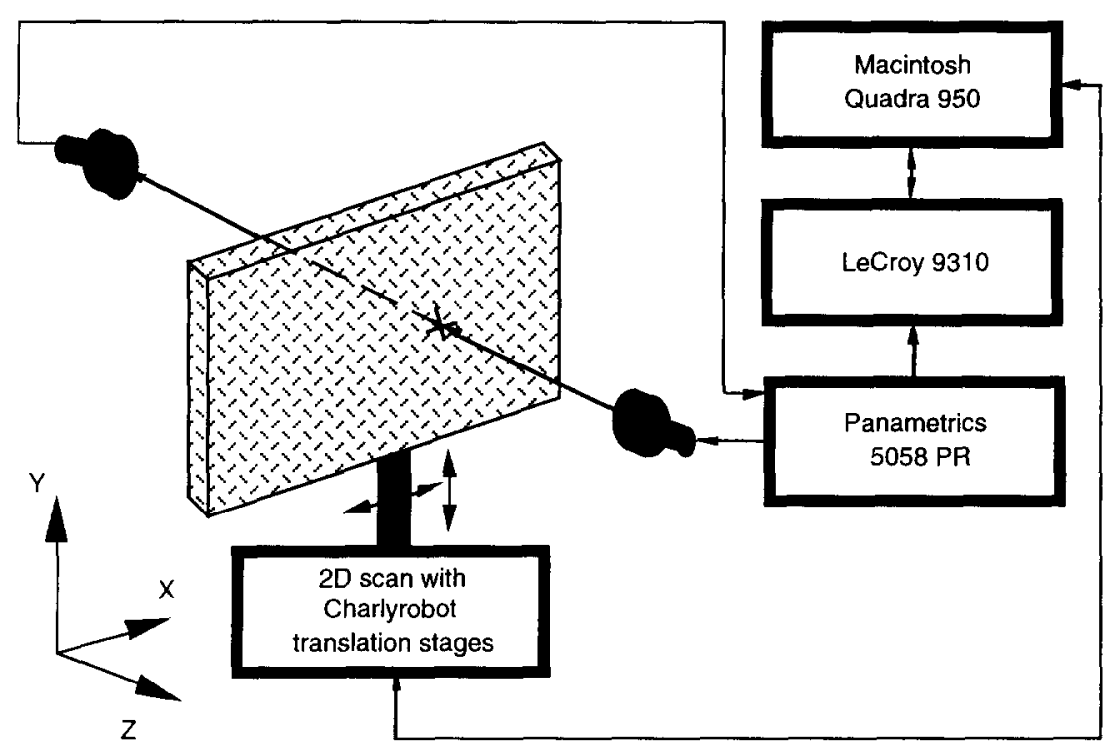

Fig. 5. Schematic of the experimental system for the transmission configuration and two-dimensional scan.

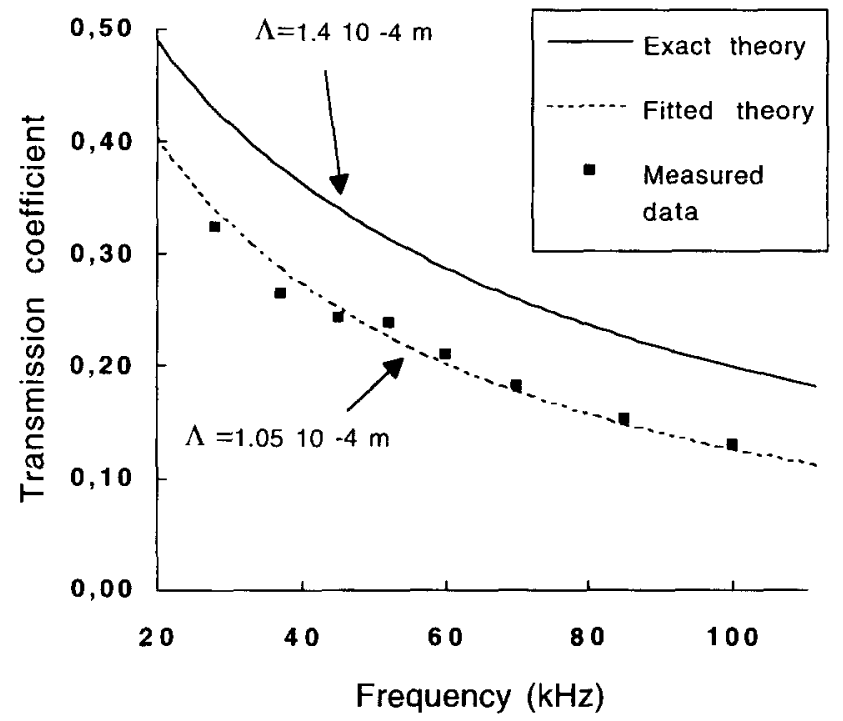

Fig. 6. Transmission coefficient versus frequency. The predicted values (-__ ) are obtained with $A=1.4 \times 10^{-4} \mathrm{~m}$, while those with the linear regression $(--)$ yiclds $A=1.05 \times 10^{-4} \mathrm{~m}$. The parameter $A^{\prime}$ is determined with the BET method $[8,9]$.

part of the acoustical propagation $n(\omega)$ index as

$n_{\mathrm{r}}=\operatorname{Re}\left(\frac{c_{0}}{c(\omega)}\right)=\operatorname{Re}(\sqrt{\alpha(\omega) \beta(\omega)})$,

where $c_{0}$ and $c(\omega)$ are, respectively, the phase wavespeed of sound in air and in the fluid saturating the material. When frequency tends to infinity, the squared propagation index is equal to

$\left(n_{\mathrm{r}}\right)^{2}=\alpha_{\infty}\left[1+\delta\left(\frac{1}{\Lambda}+\frac{\gamma-1}{B A^{\prime}}\right)\right]$

When the measurement of the propagation index is done at sufficiently high frequency (where $\delta$ tends to zero), the value of the tortuosity is then given by a simple wave speed measurement.

Additionally, due to dispersion, the dynamic tortuosity is frequency dependent. This feature is well documented for water-saturated media such as rocks or ceramics [21-26]. These measurements make use of a routine based on the phase spectrum [27]. The influence of dispersion can be drastic in some cases with watersaturated samples. $\Lambda$ ir-saturated porous materials, however, exhibit slight dispersion [28]. Consequently, the change of the tortuosity with frequency is generally small. Some characteristic results obtained with a reticulated plastic foam of the Tramico type are given in Table 1 . The results along two principal directions, i.e. the out-of-plane direction which is along the axis normal to the surface of the sample (axis 3 ), and the in-plane direction (axis 1) which lies in the plane of the sample, are different. The material is assumed to be transversely isotropic or having hexagonal symmetry, meaning in turn that the in-plane properties are isotropic. Then the Fresnel relation can be obtained by using a reasoning

Table 1

Values of the coefficient of tortuosity for the Tramico plastic foam at various frequencies, along two principal directions (in-plane and outof-plane)

\begin{tabular}{lll}
\hline Frequency $(\mathrm{kHz})$ & Tortuosity (out-of-plane) & Tortuosity (in-plane) \\
\hline 40 & 1.23 & 1.33 \\
75 & 1.21 & 1.32 \\
180 & 1.20 & 1.31 \\
$\alpha_{\infty}$ & 1.17 & 1.30 \\
\hline
\end{tabular}


similar to that used in crystal optics.

$\frac{1}{n_{\mathrm{r}}^{2}} \equiv\left[\operatorname{Re}\left(\frac{c(\omega)}{c_{0}}\right)\right]^{2}=\frac{\cos ^{2} \theta}{n_{z \mathrm{r}}^{2}}+\frac{\sin ^{2} \theta}{n_{x \mathrm{r}}^{2}}$.

Let us consider a monochromatic plane wave propagated along the direction of the unit wave normal $\boldsymbol{w}$, i.e. described by the factor

$\exp \left[j \omega\left(\frac{n(\omega)}{c_{0}} \boldsymbol{r} \cdot \boldsymbol{w}-t\right)\right]$

Substituting this dependence in Eqs. (11) and (2) we obtain

$\rho_{0} \tilde{\boldsymbol{\alpha}}_{i j}(\omega) v_{j}=\frac{n(\omega)}{c_{0}} w_{i} p ; \quad \frac{1}{K_{\mathrm{a}}} \beta(\omega) p=\frac{n(\omega)}{c_{0}} w_{i} v_{i}$.

Eliminating $p$ in Eq. (26) and making use of coordinate axes coincident with the principal axes of the tensor $\tilde{\boldsymbol{\alpha}}_{i j}(\omega)$ gives the three equations:

$v_{i}=\frac{n^{2}}{\beta x_{i}} w_{i} \cdot \boldsymbol{w} \cdot v \quad(i=x, y, z)$.

Multiplying Eq. (27) by $w_{i}$ and adding the three resulting equations we obtain

$\frac{1}{n^{2}}=\frac{w_{x}^{2}}{n_{x}^{2}}+\frac{w_{y}^{2}}{n_{y}^{2}}+\frac{w_{z}^{2}}{n_{z}^{2}}$

where $n_{i}^{2}(i=x, y, z)$ have been defined by analogy with the isotropic case, such that $n_{i}^{2}=\alpha_{i} \beta$. Let us now consider the above complex index which assumes the form $n=n_{\mathrm{r}}(1+j \zeta)$, where $\zeta \ll 1$, and similar expressions for each of the $n_{i}$. In fact, based on the Johnson et al. [10] and Allard-Champoux [13] high frequency limits for $x$ and $\beta$, typical values for our materials are $\zeta \simeq 0.05$ at $40 \mathrm{kHz}$. Thus from Eq. (28) and the definition of the phase velocity $c(\omega)$ we obtain

$\frac{1}{n_{\mathrm{r}}^{2}} \equiv\left[\operatorname{Re}\left(\frac{c(w)}{c_{0}}\right)\right]^{2}=-\frac{w_{x}^{2}}{n_{\mathrm{r} x}^{2}}+\frac{w_{y}^{2}}{n_{\mathrm{r} y}^{2}}+\frac{w_{z}^{2}}{n_{\mathrm{r} z}^{2}}+o\left(\epsilon^{2}\right)$.

In other words, the principal tortuosities being the intersection of the ellipse with the principal axes. A very simple but efficient inversion scheme can be implemented which makes use of a linear regression routine, in order to determine the principal tortuosities from measurements of the tortuosity tensor performed along numerous nonprincipal directions of propagation. The recovered values can then be directly compared to those contained in Table 1.

Experiments were carried out on a large panel $(0.3 \times 0.4 \mathrm{~m})$ of a $50 \mathrm{~mm}$ thick layer of a reticulated plastic foam of the Tramico type. The measurements were performed by using a reflecto-refractometer. The configuration of the instrument which has been used in

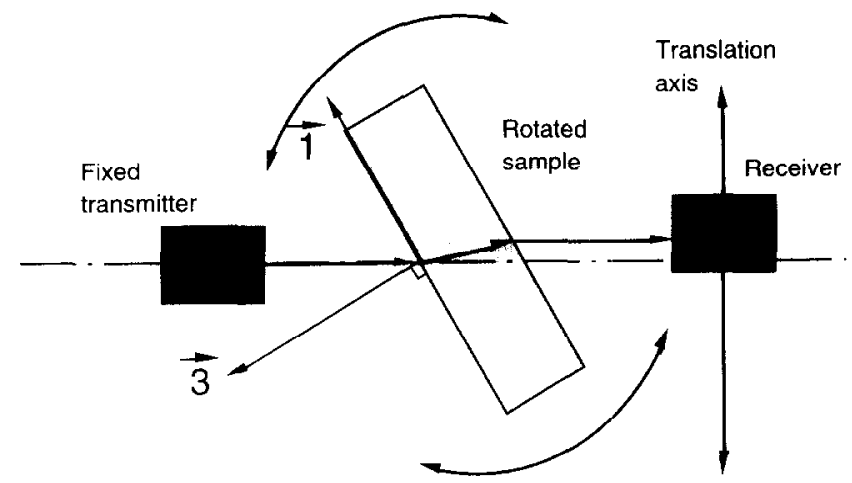

Fig. 7. Schematic diagram of the experimental set-up in the transmission configuration.

the present work is outlined in Fig. 7. The experimental apparatus has been described in Section 4. A pair of $40 \mathrm{kHz}$ narrow-band piezoelectric transducers insonified the sample. The phase wavespeed measurements were performed in the Fourier domain by using a standard intercorrelation routine [29]. A precision in the order of $0.1 \%$ was achieved. Due to the spatial shift that occurs when the ultrasonic beam crosses the sample at nonzero incidence Snell-Descartes' law of refraction is used in order to position the receiving transducer.

A polar diagram of the tortuosity in a Tramico airsaturated sample is shown in Fig. 8. The agreement between theory and experimental data is quite acceptable. The recovered principal tortuosities, i.e. $\alpha_{\infty 3}=$ $1.22 \pm 0.01$ and $x_{\infty 1}=1.34 \pm 0.01$, are within $1 \%$ of measurements done at normal incidence on samples cut along in-plane and out-of-plane principal directions, as given in Table 1. It should be pointed out that the small

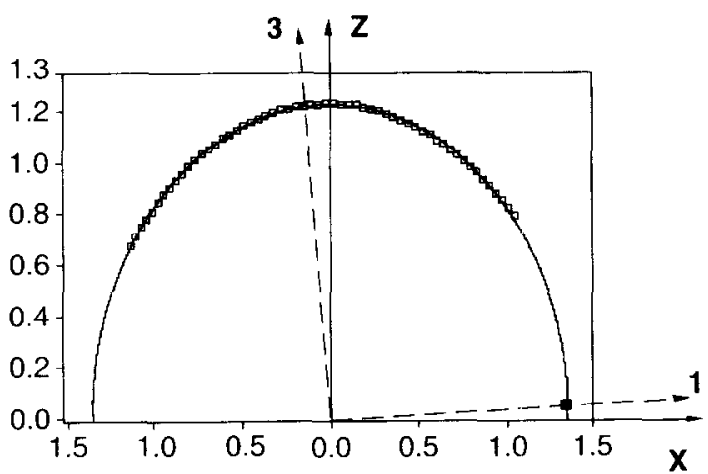

Fig. 8. Polar plot of the tortuosity versus transmission angle for a reticulated plastic foam (Tramico type). Comparison between theory (solid line) and experimental data ( $\square$ ). Axes $\overrightarrow{1}$ and $\overrightarrow{3}$, respectively, are the in-plane and out-of-plane (i.e. the normal to the surface of the plate) geometrical axes of the sample. The principal acoustical axes of the sample, which are almost aligned with axes $\overrightarrow{1}$ and $\overrightarrow{3}$, are shown with dashed lines. There is a 4 difference between both sets of axes. The recovered principal tortuosities are $\alpha_{\infty, 3}=1.22 \pm 0.01$ and $\alpha_{\infty 1}=1.34 \pm 0.01$. The black square corresponds to a measurement done at normal incidence along principal axis 1 on a sample cut from the same thick plate, as shown in Table $1\left(\alpha_{\alpha_{\alpha 1}}=1.33 \pm 0.01\right)$. 
discrepancy between the two sets of values might be due to the fact that the measurements were not carried out exactly in the same areas of the sample. An interesting finding is the fact that the principal acoustic axes of the sample do not coincide with the geometrical ones [30] (i.e. along the normal to the sample and in the plane). The inversion routine done on the tortuosity predicts a $4^{\circ}$ difference on the tortuosity, as shown in Fig. 8. Such symmetry is also observed in the measurements of the amplitude of the transmitted signal as shown in Fig. 9, its maximum being shifted by $9^{\circ}$. Consequently, our technique enables the orientation of the principal acoustical axes inside the sample to be determined. Obviously, there might exist additional Euler angles (up to two) [30] when principal axes are oriented in totally arbitrary directions. In such a case, one needs to probe various nonprincipal planes of propagation in order to recover the oricntations of the principal axcs. Such inversion routines have not been implemented so far. Interestingly, we have observed other results where the angular parallax is more pronounced (in the range of $10-20^{\circ}$ ).

Some porous materials are almost isotropic. An example is provided for a fibrous glass wool in Fig. 10, where the tortuosity versus angle is a circle. The recovered principal tortuosities are $\alpha_{\infty 3}=\alpha_{\infty 1}=1.05 \pm 0.005$. This result is also a general check of the implementation of the signal processing and data analysis schemes. It should be emphasized that this porous medium has a tortuosity close to 1 . In fact, when the tortuosity is exactly equal to 1 , there cannot exist any anisotropy. Consequently, one can expect significant anisotropy effects for porous media having large tortuosity (in the range of 2-3).

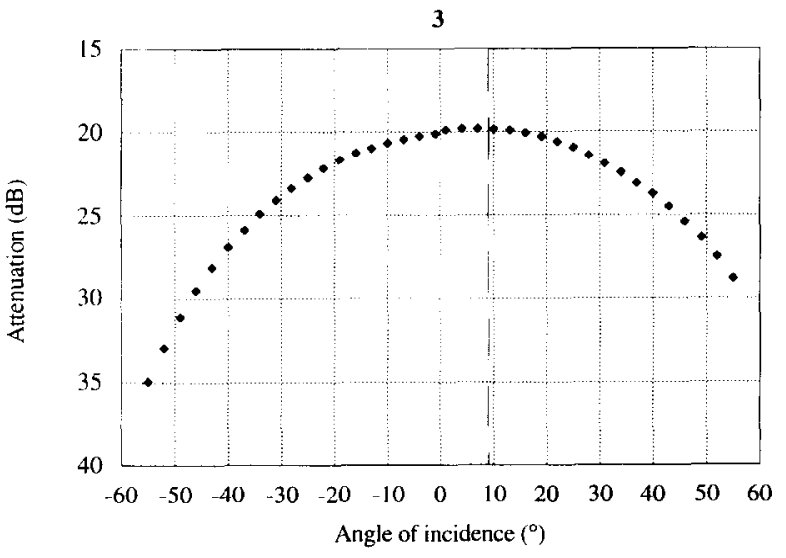

Fig. 9. Plot of the amplitude attenuation versus incidence angle for the reticulated plastic foain (Tramico type) studied in Fig. 2. The vertical solid line (along $0^{\circ}$ ) shows the normal to the sample (i.e. axis 3 ). The dashed line represents the angle of incidence corresponding to the maximum of the transmitted amplitude. There is a $9^{\circ}$ shift from axis $\overrightarrow{3}$.
3

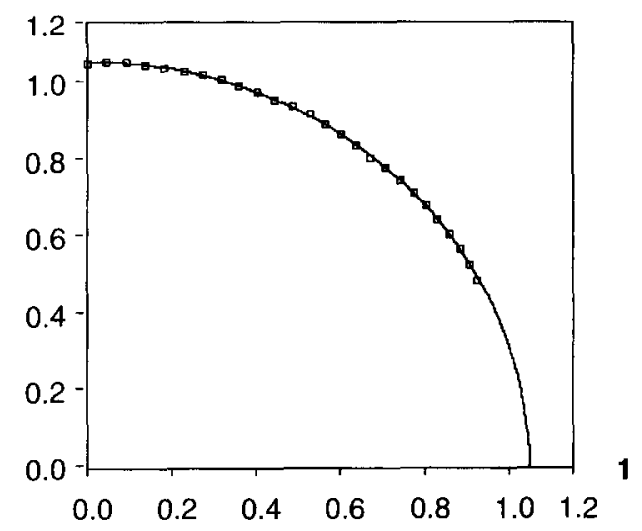

Fig. 10. Polar plot of the tortuosity versus transmission angle for a fibrous glass wool. Comparison between theory (solid line) and experimental data (as shown with blank squares). Axes $\overrightarrow{1}$ and $\overrightarrow{3}$ have the same meaning as in Fig. 8. The recovered principal tortuosities are $\alpha_{\infty<3}=\alpha_{\infty 11}=1.05 \pm 0.005$.

\section{Review of Biot theory}

More than 40 years ago, M.A. Biot [31-34] did propose a semi-phenomenological theory which provides a rigorous description of the propagation of acoustical waves in porous media saturated by a compressible viscous fluid. Such diphasic materials are supposed to be elastic and homogeneous. This theory derives the equations of motion of each phase (i.e. the solid frame and the fluid) based on energy considerations which include the inertial, the potential and the viscous coupling between the two phases. For an isotropic porous medium, three different bulk modes are predicted, i.e. two compression waves and one shear wave. One compression wave, the so-called wave of the first type or fast longitudinal wave, and the transverse wave are similar to the two bulk waves observed in an isotropic linear elastic solid. The other longitudinal wave, called a wave of the second kind, or slow wave, is a highly damped and very dispersive mode. It is diffusive at low frequencies and propagative at high frequencies. It has been observed for the first time by Plona in 1980 [35] and in subsequent years by others [36-38]. Others workers (e.g. Van Dongen et al. [39]) have studied the propagation of wcak shock wave in permcable foams with the linear Biot theory. Biot theory was initially introduced for petroleum prospection and research. Due to its very general and rather fundamental character, it has been applied in various fields of acoustics such as geophysics, underwater acoustics, sismology, etc.

A short review of the Biot theory is provided next. To derive the equations of motion for both phases, one needs to first evaluate the various energy terms, i.e. potential, kinetic and dissipative. If $u$ and $U$ represent the displacement fields of the solid phase (s) and of the fluid (f), and by introducing the spatial derivative 
operator $L_{i j}$, the three energy terms can be written in the following form:

$\left[L_{i j}\right]^{\top}=\left[\begin{array}{cccccc}\frac{\partial}{\partial x} & 0 & 0 & \frac{\partial}{\partial x} & 0 & \frac{\partial}{\partial z} \\ 0 & \frac{\partial}{\partial y} & 0 & \frac{\partial}{\partial x} & \frac{\partial}{\partial y} & 0 \\ 0 & 0 & \frac{\partial}{\partial z} & 0 & \frac{\partial}{\partial y} & \frac{\partial}{\partial z}\end{array}\right]$,

$E_{\mathrm{P}}=\frac{1}{2}\left(\epsilon_{i j}^{\mathrm{T}} C_{i j k l} \epsilon_{k l}+R e^{2}+2 \epsilon_{i j}^{\mathrm{T}} Q_{i j} \delta_{i j} e\right)$,

$E_{\mathrm{C}}=\frac{1}{2}\left(\rho_{11} \dot{u}^{\mathrm{T}} \dot{u}+2 \rho_{12} \dot{u}^{\mathrm{T}} \dot{U}+\rho_{22} \dot{U}^{\mathrm{T}} \dot{U}\right)$,

$E_{\mathrm{D}}=\frac{1}{2} b(\omega)(\dot{u}-\dot{U})^{2}$, with $b(\omega)=\phi^{2} \frac{\eta}{k_{0}} \sqrt{1+\frac{M}{2} \mathrm{j} X}$,

where the function $X(\omega)$ was expressed in Section 2 by Eq. (6). The densities $\rho_{11}$ and $\rho_{22}$ and the inertial coupling term $\rho_{12}$ are linked to the actual density of the solid frame $\rho_{\mathrm{s}}$ and those of the fluid $\rho_{\mathrm{f}}$ by the following relationships:

$\rho_{12}=-\left(\alpha_{x},-1\right) \phi \rho_{\mathrm{f}}$,

$\rho_{11}=(1-\phi) \rho_{\mathrm{s}}-\rho_{12}$,

$\rho_{22}=\phi \rho_{\mathrm{f}}-\rho_{12}$,

$\rho_{11} \rho_{22}-\rho_{12}^{2}=0$,

where $\phi$ and $\alpha_{\infty}$, respectively, are the porosity and tortuosity (as introduced in this review in Section 2). The next step is to utilize the Lagrange formalism to derive the equations of motion for both phases:

$\frac{\mathrm{d}}{\mathrm{d} t}\left(\frac{\partial E_{\mathrm{C}}}{\partial \dot{u}}\right)+\frac{\partial E_{\mathrm{p}}}{\partial u}+\frac{\partial E_{\mathrm{D}}}{\partial \dot{u}}=0$.

The same equation is also written by replacing $u$ by $U$ for the fluid. By using Eqs. (31) (34) for the three energy terms, and with the help of the $L$ operator, as given in Eq. (30), one finally obtains

$L_{i j}^{\mathrm{T}} C_{i j k l} L_{k l} u+L_{i j}^{\mathrm{T}} Q_{i j} \delta_{k l} L_{k l} U-\omega^{2}\left(\tilde{\rho}_{11} u+\tilde{\rho}_{12} U\right)=0$,

$L_{i j}^{\mathrm{T}} Q_{k l} \partial_{k l} L_{i j} u+L_{i j}^{\mathrm{T}} R L_{i j} U-\omega^{2}\left(\tilde{\rho}_{12} u+\tilde{\rho}_{22} U\right)=0$,

where the tilde densities are linked to the nontilde ones through the damping function $b(\omega)$ as expressed by the relationships (each density is consequently complex, the imaginary part being responsible for damping due to viscous effects):

$$
\tilde{\rho}_{11}=\rho_{11}+j \frac{b(\omega)}{\omega} ; \tilde{\rho}_{22}=\rho_{22}+j \frac{b(\omega)}{\omega} ; \tilde{\rho}_{12}=\rho_{12}-j \frac{b(\omega)}{\omega} .
$$

When limiting the analysis to the isotropic case, the stiffness matrix of a porous frame is given as the usual expressions for a linear elastic solid:

$\left[C_{I J}\right]=\left[\begin{array}{cccccc}A+2 N & A & A & 0 & 0 & 0 \\ A & A+2 N & A & 0 & 0 & 0 \\ A & A & A+2 N & 0 & 0 & 0 \\ 0 & 0 & 0 & N & 0 & 0 \\ 0 & 0 & 0 & 0 & N & 0 \\ 0 & 0 & 0 & 0 & 0 & N\end{array}\right]$

where the rigidities are given by

$$
\begin{aligned}
& N=E\left(1+\mu_{\mathrm{s}}\right) / 2(1+v), \\
& A=\left[(1-\phi)^{2} / \phi\right] K_{\mathrm{e}}(\omega)+[2 N v /(1-2 v)], \\
& R=\phi K_{\mathrm{e}}(\omega), \quad Q=(1-\phi) K_{\mathrm{e}}(\omega),
\end{aligned}
$$

where $\mu_{\mathrm{s}}$ is the structural damping coefficient of the frame and $K_{\mathrm{e}}(\omega)=K_{\mathrm{a}} / \beta(\omega)$. These coefficients reduce to the usual Lamé parameters of a linear elastic solid $\lambda$ and $\mu$ as expressed from the Young modulus $E$, and the Poisson ratio $v$, just by putting the porosity equal to 1 $(\phi=1)$. Next, for the case of isotropic porous media, the plane wave solution of Eqs. (36) and (37) is studied with the customary scalar and vector potential decomposition, respectively, for the compression and shear waves. For the case of the longitudinal wave this can be written down as $u=V \varphi^{\mathrm{s}} ; \mathrm{U}=\boldsymbol{V} \varphi^{\mathrm{f}}$, with $\boldsymbol{V}=[\partial / \partial x, \partial / \partial y, \partial / \partial z]$. This yields

$\left(\nabla^{2}-()^{2}\left[\begin{array}{ll}P & Q \\ Q & R\end{array}\right]^{-1}\left[\begin{array}{cc}\tilde{\rho}_{11} & \tilde{\rho}_{12} \\ \tilde{\rho}_{12} & \tilde{\rho}_{22}\end{array}\right]\right)\left(\begin{array}{l}\varphi^{\mathrm{s}} \\ \varphi^{\mathrm{f}}\end{array}\right)=\left(\begin{array}{l}0 \\ 0\end{array}\right)$,

where $P=A+2 N$, providing:

$k_{1.2}^{2}=\frac{\omega^{2}}{2\left(P R-Q^{2}\right)}\left(P \tilde{\rho}_{22}+R \tilde{\rho}_{11}-2 Q \tilde{\rho}_{12} \pm \sqrt{\Delta}\right)$,

with: $\Delta=\left(P \tilde{\rho}_{22}+R \tilde{\rho}_{11}-2 Q \tilde{\rho}_{12}\right)^{2}$

$$
-4\left(P R-Q^{2}\right)\left(\tilde{\rho}_{11} \tilde{\rho}_{22}-\tilde{\rho}_{12}^{2}\right)
$$

where the two wavevector solutions 1 and 2, respectively, are given by the + and - signs in Eq. (43). From these expressions, one can then obtain the ratio between the displacement amplitude in the fluid to the one of the 
solid frame:

$A_{i}=\frac{\varphi_{i}^{\mathrm{f}}}{\varphi_{i}^{\mathrm{s}}}=\frac{P k_{i}^{2}-\omega^{2} \tilde{\rho}_{11}}{\omega^{2} \tilde{\rho}_{12}-Q k_{i}^{2}}$.

For the transverse wave the calculations proceed in a similar way, by replacing the gradient operator by a curl, i.e. $u^{\mathrm{s}}=\nabla \times \psi^{\mathrm{s}} ; U^{\mathrm{f}}=\nabla \times \psi^{\mathrm{f}}$, and yields

$N V^{2} \psi^{\mathrm{s}}+\omega^{2} \tilde{\rho}_{11} \psi^{\mathrm{s}}+\omega^{2} \tilde{\rho}_{12} \psi^{\mathrm{f}}=0$,

$\omega^{2} \tilde{\rho}_{12} \psi^{\mathrm{s}}+\omega^{2} \tilde{\rho}_{22} \psi^{\mathrm{f}}=0$,

$k_{3}^{2}-\frac{\omega^{2}}{N} \frac{\left(\tilde{\rho}_{11} \tilde{\rho}_{22}-\tilde{\rho}_{12}^{2}\right)}{\tilde{\rho}_{22}} ; \quad A_{3}=\frac{\psi_{3}^{\mathrm{f}}}{\psi_{3}^{\mathrm{s}}}=-\frac{\tilde{\rho}_{12}}{\tilde{\rho}_{22}}$.

When dealing with anisotropic porous materials, one starts from the linear elasticity behavior equation written down in tensorial form:

$\left[\begin{array}{c}\sigma_{i j} \\ s\end{array}\right]=\left[\begin{array}{cc}C_{i j k l} & Q_{i j} \delta_{i j} \\ Q_{k l} \delta_{k l} & R\end{array}\right]\left[\begin{array}{c}\epsilon_{k l} \\ e\end{array}\right]$,

where $i, j, k, l=1,2,3$, and where $\sigma_{i j}$ and $s$ represent the stresses, respectively, in the solid and in the fluid, $\epsilon_{k l}$ and $e$ being the corresponding strains. The elasticity tensor is denoted by $C_{i j k l}$, the compressibility modulus of the fluid by $R$, and $Q_{i j}$ is the diagonal coupling tensor which is of the potential type Biot himself did study anisotropy of porous solids [32] and one can find the coupled equations of motion in that reference. More recently various authors have revisited the anisotropic Biot theory, see for example Ref. [40].

\section{Characteristic surfaces of the anisotropic Biot waves}

From Eq. (48) it is then possible to derive the equations of motion, respectively, of the solid and fluid phases which are written as

$$
\begin{aligned}
& \rho_{11} \frac{\partial^{2} u_{i}}{\partial t^{2}}+\rho_{12} \frac{\partial^{2} U_{i}}{\partial t^{2}}=C_{i j k l} \frac{\partial^{2} u_{l}}{\partial x_{j} \partial x_{k}}+Q_{j j} \frac{\partial^{2} U_{i}}{\partial x_{j}^{2}} \\
& \rho_{12} \frac{\partial^{2} u_{i}}{\partial t^{2}}+\rho_{22} \frac{\partial^{2} U_{i}}{\partial t^{2}}=Q_{j j} \frac{\partial^{2} u_{i}}{\partial x_{j}^{2}}+R \frac{\partial^{2} U_{l}}{\partial x_{i} \partial x_{l}} .
\end{aligned}
$$

where the densities are kept isotropic (see previous section).

The next step deals with searching solutions in the form of plane waves. Adding dissipative terms to Eq. (49) results in introducing complex densities (see Eq. (38)). In such a case, the wavespeeds are complex and dispersive (cf. Section 7).

In this work we have developed routines, based on the standard Biot model, which compute the various characteristic surfaces (slowness, phase and group velocities) in a given plane for anisotropic porous media. By increasing the weight of the coupling constants, one can continuously vary from the limit case of anisotropic solids with no porosity to a number of different porous media. When the coupling terms are not equal to zero, one finds four instead of three propagating modes, the two other eigenvalues being complex, meaning in turn that the two remaining solutions do correspond to some evanescent modes. The slow transverse mode is unchanged. The fourth mode often called the Biot mode [35-38], which is very similar to the pseudo-thermal wave (see Section 10 for further details) observed in dynamic thermoelasticity, has always a very small wavespeed and is very highly damped.

We restrict here to the simplier case of real densities, which is, however, sufficient to describe the main features related to anisotropy. Writing down the calculations yields the following compatibility equation:

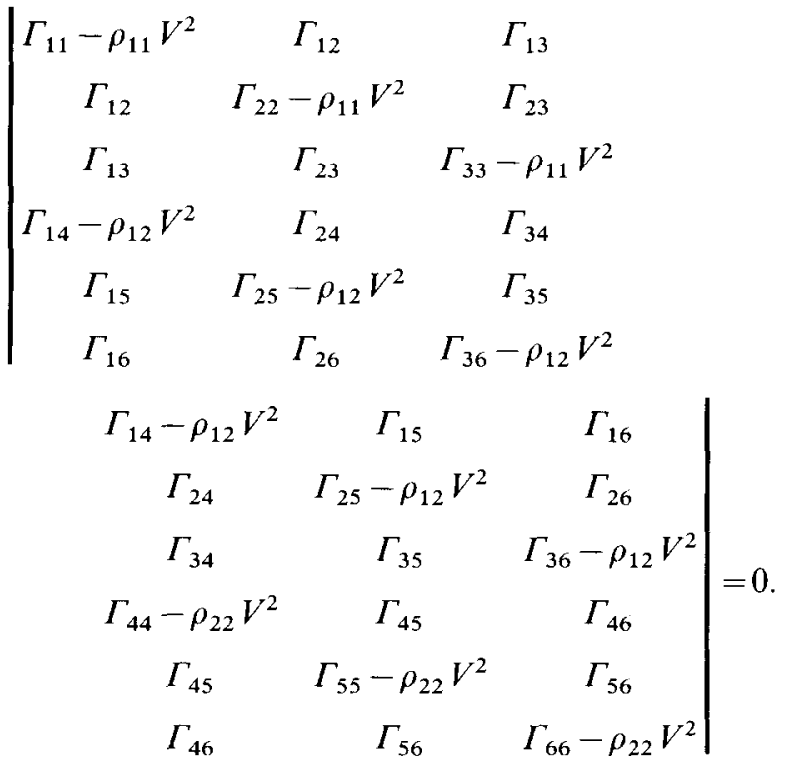

In such an equation, $V$ represents the phase wavespeed $(V=\omega / k)$, and the terms $\Gamma_{i l}$ are linked to $C_{i j k l}, R$ and $Q_{i j}$. For instance, the $I$ ' components of the top left $3 \times 3$ sub-determinant is nothing else than the Christoffel tensor, i.e. $\Gamma_{i l}=C_{i j k l} w_{j} w_{k}$, where $i, j, k, l=1,2,3$, where $\boldsymbol{w}$ is the unit vector along the direction of propagation. The full determinant has four real roots corresponding to propagating modes as well as two additional imaginary roots which do not contributc to the propagation. By supposing the propagation to take place in plane $(1,3)$ of a transverse isotropic solid where $w_{1}=\sin \theta, w_{2}=0$ and $w_{3}=\cos \theta$, then the above determinant has the following simplier form:

$$
\begin{aligned}
& V^{2}\left[\left(\rho_{11} \rho_{22}-\rho_{12}^{2}\right) V^{2}-\rho_{22} \Gamma_{22}\right] \\
& \times\left|\begin{array}{cccc}
\Gamma_{11}-\rho_{11} V^{2} & \Gamma_{13} & \Gamma_{14}-\rho_{12} V^{2} & \Gamma_{16} \\
\Gamma_{13} & \Gamma_{33}-\rho_{11} V^{2} & \Gamma_{34} & \Gamma_{36}-\rho_{12} V^{2} \\
\Gamma_{14}-\rho_{12} V^{2} & \Gamma_{34} & \Gamma_{44}-\rho_{22} V^{2} & \Gamma_{46} \\
\Gamma_{16} & \Gamma_{36}-\rho_{12} V^{2} & \Gamma_{46} & \Gamma_{66}-\rho_{22} V^{2}
\end{array}\right|=0,
\end{aligned}
$$


where

$$
\begin{aligned}
& \Gamma_{11}=(A+2 N) w_{1}^{2}+L w_{3}^{2} ; \Gamma_{14}=M w_{1}^{2} ; \Gamma_{16}=M w_{1} w_{3}, \\
& \Gamma_{22}-N w_{1}^{2}+L w_{3}^{2} ; \Gamma_{34}=Q w_{1} w_{3} ; \Gamma_{36}=Q w_{3}^{2}, \\
& \Gamma_{33}=L w_{1}^{2}+C w_{3}^{2} ; \Gamma_{44}=R w_{1}^{2} ; \Gamma_{46}=R w_{1} w_{3}, \\
& \Gamma_{13}=(F+L) w_{1} w_{3} ; \Gamma_{66}=R w_{3}^{2},
\end{aligned}
$$

with $A+2 N=C_{11}=C_{22}, A=C_{12}, F=C_{13}, C=C_{33}, L=$ $C_{44}=C_{55}, \quad N=C_{66}, \quad M=Q_{11}=Q_{22}, \quad$ and $\quad Q=Q_{33}$. Accordingly, there exists a trivial root, i.e. $V=\sqrt{\rho_{22} \Gamma_{22} /\left(\rho_{11} \rho_{22}-\rho_{12}^{2}\right)}$, which is identical to $\sqrt{\Gamma_{22} / \rho_{11}}$ when $\rho_{12}=0$, which is the velocity of a $\mathrm{SH}$ type wave. Some significant numerical results obtained with the above procedure are plotted on Fig. 11. Further details are provided in Ref. [42], or elsewhere [43].

\section{Transmission coefficient for a Biot anisotropic porous layer}

In this section, the problem of a plane wave impinging a fluid-porous medium plane interface with an oblique angle of incidence $\theta$ is considered. The porous material is supposed to belong to hexagonal symmetry. It is described in the frame of Biot theory. Solutions for the reflected and refracted waves which verify the Biot equations of motion are sought. In order to solve this problem the wavespeeds (cf. Section 8) and the polarizations of the various modes [44], which are propagated in the porous medium, are required. For that purpose, the classical boundary conditions introduced by Deresiewicz and Rice [45] and Rosenbaum [46] are used. The reflection and transmission coefficients are then calculated by using the Poynting theorem as applied to acoustics $[41,47]$. Numerical simulations are designed to obtain these coefficients versus the angle of incidence of the incident wave.

The propagation is done in plane $x z$, where $x$ lies at the surface of the material while $z$ is along the thickness. The fluid fills the negative half space $(z<0)$ and the porous material extends on the other half space $(z>0)$. The angle of incidence of the wave in the fluid, at the interface $z=0$, is denoted $\theta$, as shown in Fig. 12. Due to mode conversion at the interface, there exists one reflected and three refracted waves. It should be noted that the fourth transmitted wave, whose polarization is along (or close to for a quasi-transverse wave) the axis orthogonal to the propagation plane is the second shear wave. This mode does not exist for the geometry considered here because it cannot respect the continuity of the displacement field as the polarization of the incident wave is in plane $x z$ and accordingly does not have a component along $y$. Due to anisotropy the polarization of the various reflected and transmitted modes are not generally pure, but instead quasi-longitudinal or quasi-
3

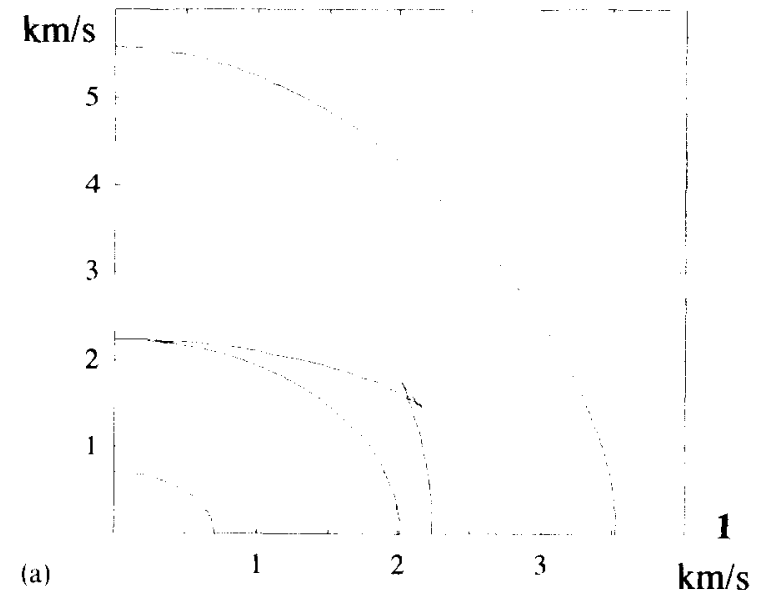

3

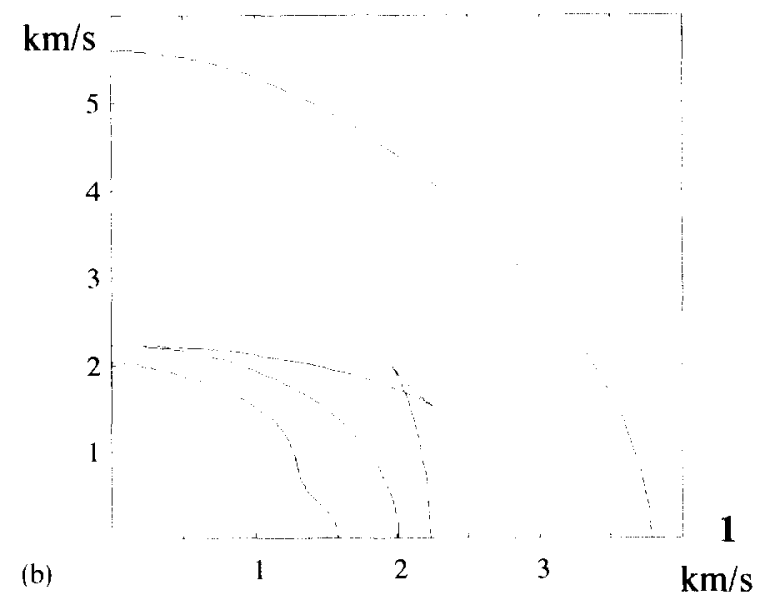

Fig. 11. Characteristic surfaces (wavespeed curves) in plane $(1,3)$ of a transversely isotropic porous material. The properties of the material are the following: $C_{11}=26.1 \mathrm{GPa} ; C_{3.3}=65.5 \mathrm{GPa}: C_{44}=10.5 \mathrm{GPa}$; $C_{66}=8.50 \mathrm{GPa} ; \quad C_{13}=9.58 \mathrm{GPa} ; \quad C_{12}=C_{11}-2 C_{66}=9.31 \mathrm{GPa} ; \rho_{11}=$ $2.108 \mathrm{~g} \mathrm{~cm}^{-3} ; \rho_{22}=1 \mathrm{~g} \mathrm{~cm}^{-3} ; \rho_{12}=0.1 \mathrm{~g} \mathrm{~cm}^{-3}$. (a) $M=Q=R=0.5 \mathrm{SI}$ : (b) $M=Q=7.5 \mathrm{SI}$; $R=5 \mathrm{SI}$. The solid lines represent the group wavespeeds. The slow transverse mode nearly has identical phase and group wavespeeds.

transverse. However, the Snell-Descartes law of refraction is verified in this case. It can be stated in the form

$\frac{\omega}{c_{0}} \sin \theta=\frac{\omega}{v_{i}} \sin \alpha_{i}=\sigma$,

where $c_{0}$ is the incident wavespeed and $v_{i}$ is the velocity of the transmitted fast $(i=1)$ and slow $(i=3)$ quasilongitudinal waves and quasi-transverse $(i=2)$ waves. The quantity $\alpha_{i}$ corresponds to the refraction angles. The viscous damping effects are neglected here, but they can also be included in the treatment. Their influence does not modify the structure of the above equations, as was stated in Section 8. They can simply be taken into account by adding an imaginary part in the $\rho_{12}$ 


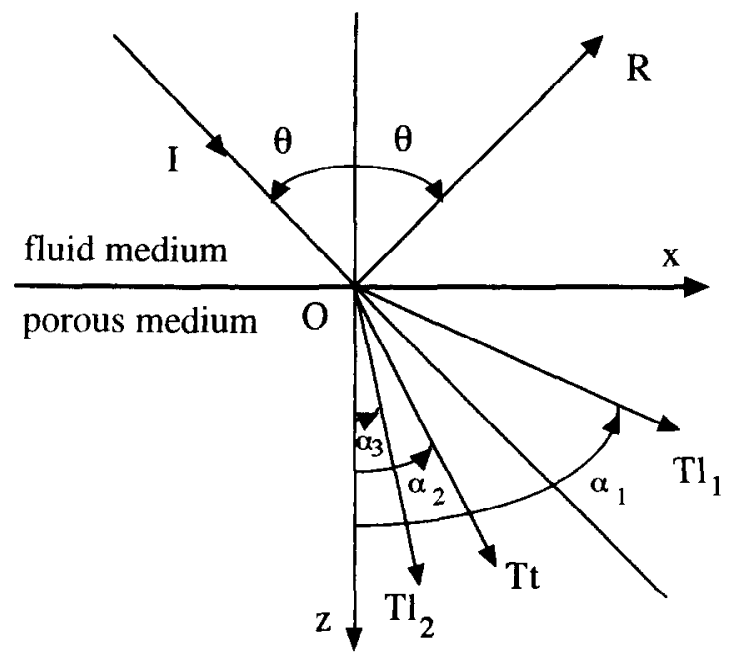

Fig. 12. Geometry of the problem. I, incident compressional wave; R, reflected wave; $T 1_{1}$, transmitted fast compressional wave; $\mathrm{Tl}_{2}$, transmitted slow compressional wave; $T_{\mathrm{t}}$, transmitted shear wave.

coupling density $[10,37]$. The boundary conditions for this problem are written as follows:

(1) Continuity of the normal stress

$\left.\left(\sigma_{z z}+s\right)\right|_{z=0^{+}}=-\left.p\right|_{z=0^{-}}$.

(2) Continuity of the shear stress

$\left.\sigma_{x z}\right|_{z-0^{+}}=0$.

(3) Continuity of the normal particle velocity

$\left.(1-\phi) \dot{u}_{z}\right|_{z=0^{+}}+\left.\phi \dot{U}_{z}\right|_{z=0^{+}}=\left.\dot{U}_{z}^{\prime}\right|_{z=0^{-}}$.

(4) Continuity of the fluid pressure

$\left.s\right|_{z=0^{+}}=-\left.\phi p\right|_{z=0^{-}}$.

The expansion of the above four equations yields the following set of equations:

$$
\begin{aligned}
& \left.(F+M) \frac{\partial u_{x}}{\partial x}\right|_{z=0^{+}}+\left.(C+Q) \frac{\partial u_{z}}{\partial z}\right|_{z=0^{+}} \\
& +\left.(Q+R)\left(\frac{\partial U_{x}}{\partial x}+\frac{\partial U_{z}}{\partial z}\right)\right|_{z=0^{+}}=-\left.\left(p^{i}+p^{r}\right)\right|_{z=0^{-}} \\
& \left.\left(\frac{\partial u_{x}}{\partial z}+\frac{\partial u_{z}}{\partial x}\right)\right|_{z=0^{+}}=0, \\
& \left.(1-\phi) \dot{u}_{z}\right|_{z=0^{+}}+\left.\phi \dot{U}_{z}\right|_{z=0^{+}}=\left.\dot{U}_{z}^{\prime}\right|_{z=0^{-}}, \\
& \left.M \frac{\partial u_{x}}{\partial x}\right|_{z=0^{+}}+\left.Q \frac{\partial u_{z}}{\partial z}\right|_{z=0^{+}}+\left.R\left(\frac{\partial U_{x}}{\partial x}+\frac{\partial U_{z}}{\partial z}\right)\right|_{z=0^{+}} \\
& =-\left.\phi\left(p^{i}+p^{r}\right)\right|_{z=0^{-}},
\end{aligned}
$$

The displacement fields $\boldsymbol{u}$ and $\boldsymbol{U}$ for the solid and fluid phases of the porous medium are due to the contribu- tions of various modes as written in Eq. (62), while the displacement field in the external fluid is given by Eq. (63).

$$
\begin{aligned}
\boldsymbol{U}= & \sum_{i=1}^{3} C_{i} \boldsymbol{d}_{i} \exp \left[\mathrm{j}\left(\omega t-\sigma x-\gamma_{i} z\right)\right], \\
\boldsymbol{U}= & \sum_{i=1}^{3} C_{i} \boldsymbol{D}_{i} \exp \left[\mathrm{j}\left(\omega t-\sigma x-\gamma_{i} z\right)\right], \\
U^{\prime}= & \left(\begin{array}{c}
\sin \theta \\
\cos \theta
\end{array}\right) \exp \left[\mathrm{j} \omega\left(t-\frac{x \sin \theta}{c_{0}}-\frac{z \cos \theta}{c_{0}}\right)\right] \\
& +\left(\begin{array}{c}
\sin \theta \\
-\cos \theta
\end{array}\right) a_{\mathrm{r}} \exp \left[\mathrm{j} \omega\left(t-\frac{x \sin \theta}{c_{0}}-\frac{z \cos \theta}{c_{0}}\right)\right],
\end{aligned}
$$

where the amplitude of the incident wave is normalized to 1 , and those of the reflected wave is noted $a_{\mathrm{r}}, C_{i}$ are the amplitudes of the refracted waves, $\boldsymbol{d}_{\mathrm{i}}$ and $\boldsymbol{D}_{\mathrm{i}}$ are the polarization vectors of the frame and the fluid, respectively, and where $\gamma_{i}=\left(\omega / v_{i}\right) \cos \alpha_{i}$. By reporting these expressions for $\boldsymbol{u}, \boldsymbol{U}$ and $\boldsymbol{U}^{\prime}$ in Eqs. (58)-(61), we finally obtain

$$
\begin{aligned}
& \sum_{i=1}^{3} C_{i}\left[(F+M) \sigma d_{x i}+(C+Q) \gamma_{i} d_{z i}+(Q+R)\right. \\
& \left.\times\left(\sigma D_{x i}+\gamma_{i} D_{z i}\right)\right]-\rho_{\mathrm{f}} \omega c_{0} a_{\mathrm{r}}=\rho_{\mathrm{f}} \omega c_{0} \\
& \sum_{i=1}^{3} C_{i}\left[\left(\sigma d_{z i}+\gamma_{i} d_{x i}\right)\right]+0 a_{\mathrm{r}}=0 \\
& \left.\sum_{i=1}^{3} C_{i}\left[(1-\phi) d_{z i}+\phi D_{z i}\right)\right]+a_{\mathrm{r}} \cos \theta=\cos \theta \\
& \sum_{i=1}^{3} C_{i}\left[M \sigma d_{x i}+Q \gamma_{i} d_{z i}+R\left(\sigma D_{x i}+\gamma_{i} D_{z i}\right)\right]-\phi \rho_{\mathrm{f}} \omega c_{0} a_{\mathrm{r}} \\
& \quad=\phi \rho_{\mathrm{f}} \omega c_{0} .
\end{aligned}
$$

The solution of the above set of algebraic Eqs. (64)(67) enables to determine the amplitudes of the various waves. The reflection and transmission coefficients are then calculated from the ratios of the acoustic intensities of the reflected and transmitted waves to acoustic intensities of the incident wave. The intensities themselves are defined by the absolute value of the Poynting vector as given by Auld [41]. Its expression for the case of a plane wave propagated in an isotropic porous medium was given recently by $\mathrm{Wu}$ et al. [47]:

$$
\begin{aligned}
\boldsymbol{P}= & -\mathrm{j} \omega\left[\boldsymbol{e}_{x}\left(u_{x} \sigma_{x x}+u_{z} \sigma_{x z}+U_{x} s\right)\right. \\
& \left.+\boldsymbol{e}_{z}\left(u_{x} \sigma_{x z}+u_{z} \sigma_{z z}+U_{z} s\right)\right]
\end{aligned}
$$

where $e_{x}$ and $\boldsymbol{e}_{z}$ are the unit vectors, respectively, along the $x$ - and $z$-directions, and where $\sigma_{i j}$ and $s$, respectively, are the components of the stresses applied to the solid skeleton and to the fluid. The calculation of Eq. (68) for the three modes yields the corresponding acoustical intensities which are 
(a) longitudinal waves

$$
\begin{aligned}
& I_{k}=\omega C_{k}^{2}\left[\left(\sigma \mu_{1}+\gamma_{k} \mu_{2}\right)^{2}+\left(\sigma \mu_{2}+\gamma_{k} \mu_{3}\right)^{2}\right]^{1 / 2} \\
& \mu_{1}=(A+2 N) d_{x k}^{2}+L d_{z k}^{2}+2 M d_{x k} D_{x k}+R D_{x k}^{2} . \\
& \mu_{2}=(L+F) d_{x k} d_{z k}+M d_{x k} D_{z k}+Q d_{z k} D_{x k}+R D_{x k} D_{z k}, \\
& \mu_{3}=L d_{x k}^{2}+C d_{z k}^{2}+2 Q d_{z k} D_{z k}+R D_{z k}^{2},
\end{aligned}
$$

where $k=1$ for the fast longitudinal wave and $k=3$ for the slow longitudinal wave; and

(b) transverse wave

$$
\begin{aligned}
I_{2} & =\omega C_{2}^{2} \\
& \times\left\{\left[\sigma\left((A+2 N) d_{x 2}^{2}+L d_{z 2}^{2}\right)+\gamma_{2}(F+L) d_{x 2} d_{z 2}\right]^{2}\right. \\
& \left.+\left[\gamma_{2}\left((A+2 N) d_{z 2}^{2}+L d_{x 2}^{2}\right)+\sigma(F+L) d_{x 2} d_{z 2}\right]^{2}\right\}^{1 / 2} .
\end{aligned}
$$

The intermediate calculations are given in the Annex of Ref. [44]. By taking the limit case of isotropy in the above equations, one recovers quite easily the theoretical expressions obtained by $\mathrm{Wu}$ in his reference paper [47].

To numerically validate the present model, which is adequate as well for anisotropic porous media, it is possible to check the numerical results presented by $\mathrm{Wu}$ for the isotropic limit case, when considering exactly the same parameters. This has been done in Ref. [44] (i.e. see Fig. 13), where the Wu results (see Figs 3 and 4 in Ref. [47]) are exactly retrieved. The conservation of energy is numerically verified, which is a correct result for a lossless porous material. It should be emphasized that the general shape of the curves is quite similar to what is obtained for elastic or viscoelastic nonporous materials (e.g. see Refs. $[48,49]$ ). The major difference deals, for porous media, with the existence of the Biot mode (or slow longitudinal wave). The amplitude of

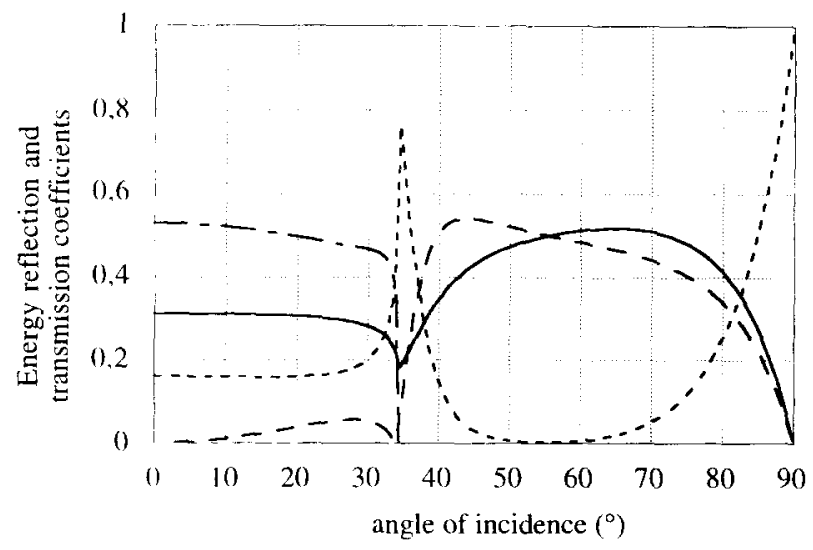

Fig. 13. Energy reflection and transmission coefficients versus angle of incidence. (- . - ) Fast compressional mode; (_- ) slow compressional mode; (- - ) shear mode; $(--)$ reflection mode. Numerical data used in the computations are: $P=10.4 \mathrm{GPa}: Q=1.1 \mathrm{GPa}: R=$ $0.8 \mathrm{GPa} ; \quad N=2.8 \mathrm{GPa} ; \quad f=5 \times 10^{5} \mathrm{~Hz} ; \quad \phi=0.38 ; \quad x_{\infty}=1.79 ; \rho_{11}=$ $1.84 \mathrm{~g} \mathrm{~cm}^{-3} ; \rho_{12}--0.3 \mathrm{~g} \mathrm{~cm}^{-3} ; \rho_{22}=0.7 \mathrm{~g} \mathrm{~cm}^{-3} ; v_{1}=2657.2 \mathrm{~m} \mathrm{~s}^{-1}$; $v_{2}=935.8 \mathrm{~m} \mathrm{~s}^{-1} ; v_{3}=1281.4 \mathrm{~m} \mathrm{~s}^{-1} ; v^{\prime}=1500 \mathrm{~m} \mathrm{~s}^{-1}$. this mode is always very small, explaining in turn why in many experimental configurations it is difficult to observe.

\section{Analogy with the anisotropic theory of thermoelasticity}

The theory of thermoelasticity when applied to static problems allows one to predict the nature and distribution of stress fields due to thermal fields [50]. This is a basic tool in manufacturing engineering involving the use of temperature gradients during fabrication processes. The application of this theory to dynamical problems is not a trivial matter, but has been extensively described, at least for isotropic solids, by several authors $[51,52]$. In its basic isotropic version the equations of thermo-clasticity are

the equation of motion

$\rho_{\mathrm{s}} \ddot{u}_{i}=\sigma_{i j . j}(i, j=1,2,3)$,

the Duhamel-Neumann equation

$\sigma_{i j}=\left\{\lambda u_{j, j}-x_{t}(3 \lambda+2 \mu)\left(T-T_{0}\right) i \dot{\delta}_{i j}+2 \mu \epsilon_{i j}\right.$

$(i, j=1,2,3)$,

- the heat conduction equation

$\kappa \nabla^{2} T=\rho_{\mathrm{s}} C_{\mathrm{v}} \dot{T}+\alpha_{\mathrm{t}}(3 \dot{\lambda}+2 \mu) T_{0} \dot{u}_{j, j}$

In these equations $\boldsymbol{u}$ is the displacement field, $\rho_{\mathrm{s}}$ the density of the material, $\sigma_{i j}$ the stress tensor, $\alpha_{1}$ the coefficient of linear expansion, $\lambda$ and $\mu$ the Lamé elastic constants, $T-T_{0}$ the difference of temperature from the ambient, $\dot{\delta}_{i j}$ the Kronecker delta, $\epsilon_{i j}$ the strain tensor, $C_{\mathrm{v}}$ the specific heat at constant volume, and $\kappa$ the thermal conductivity.

Eqs. (72) and (73) can be generalized for an anisotropic solid in the following form:

$\sigma_{i j}=C_{i j k l} \epsilon_{k l}-C_{i j k l} \alpha_{t k l}\left(T-T_{0}\right)$,

and

$\kappa_{i j} T_{. i j}=\rho_{\mathrm{s}} C_{\mathrm{v}} \dot{T}+T_{0} C_{i j k l} \alpha_{\mathrm{t} k l} \dot{\epsilon}_{i j}$,

where $C_{i j k l}$ is the stiffness tensor, $\kappa_{i j}$ the thermal conductivity tensor and $x_{t k l}$ the thermal expansion tensor.

An unphysical property of the diffusion-type heat equation [i.e. Eq. (73) or Eq. (75)] is that a sudden change in temperature made at some position in the solid will be instantly transmitted everywhere, giving rise to an infinite propagation speed. This feature was already recognized in the last century by Maxwell, and requires a modification of the Fourier law by adding a supplementary term [53,54], i.e. Eq. (75) is rewritten

$-q_{i, i}=\rho_{\mathrm{s}} C_{\mathrm{v}} \dot{T}+T_{0} C_{i j k l} \alpha_{\mathrm{t} k l} \dot{\epsilon}_{i j}$, 
and

$\tau_{i j} \dot{q}_{j}+q_{i}=-\kappa_{i j} T_{, j}$

where $\boldsymbol{q}_{i}$ is the heat flux vector, and $\tau_{i j}$ the relaxation time tensor. When $\tau_{i j}=0$, Eq. (77) reduces to the standard Fourier law, and Eq. (76) is then identical to Eq. (75). The introduction of the relaxation time tensor converts the diffusion equation into a telegraph-type equation which is no more than the Helmholtz equation with an additional diffusive term [55]. The use of the standard Fourier law instead of Eq. (77) is, however, sufficient for most problems. The introduction of the additional relaxation term is necessary to explain the origin of the second sound at very low temperature in crystals, and has been extensively reviewed by Maris [56]. In the limiting case of an isotropic solid, Eqs. (71)(73) may be rewritten after substitution of Eq. (72) into Eq. (71), in the vector form as the set of two coupled differential equations:

$\kappa \nabla^{2} T=\rho_{\mathrm{s}} C_{\mathrm{v}} \dot{T}+T_{0} \alpha_{\mathrm{t}}(3 \lambda+2 \mu) \operatorname{div} \dot{\boldsymbol{u}}$,

and

$\mu \nabla^{2} \boldsymbol{u}+(\hat{\lambda}+\mu) \operatorname{grad} \operatorname{div} \boldsymbol{u}=\alpha_{\mathrm{t}}(3 \hat{\lambda}+2 \mu) \operatorname{grad} T+\rho_{\mathrm{s}} \ddot{u}$.

By using the customary decomposition [57] of the displacement field into a curl free and a divergence free component, i.e. $\boldsymbol{u}=\boldsymbol{u}_{1}+\boldsymbol{u}_{\mathrm{t}}$, where $\operatorname{div} \boldsymbol{u}_{\mathrm{t}}=0$, and curl $u_{1}=0$, and by applying the curl operator to both sides of Eq. (79), one obtains

$\operatorname{curl}\left(\rho_{\mathrm{s}} \ddot{\boldsymbol{u}}_{\mathrm{t}}-\mu \nabla^{2} \boldsymbol{u}_{\mathrm{t}}\right)=0$,

which is nothing else than the ordinary wave equation, as the quantity between parentheses should identically cancel because its divergence is zero. In turns, this means that the transverse wavespeed is $c_{\mathrm{t}}=\left(\mu / \rho_{\mathrm{s}}\right)^{1 / 2}$. In other words, the transverse wave is uncoupled and its wavespeed is unchanged by taking into account thermoclasticity. It could also be shown $[51,58]$ that such transverse wave is undamped and undispersed. These properties are due to the fact that no dilatation $u_{j, j}$ accompanies the transverse wave.

The results are obviously quite different for the longitudinal wave. Application of the divergence operator on Eq. (79), provides a new equation:

$\operatorname{div}\left[\rho_{\mathrm{s}} \ddot{u}_{1}-(\hat{\lambda}+2 \mu) \nabla^{2} \boldsymbol{u}_{1}+\alpha_{\mathrm{t}}(3 \hat{\lambda}+2 \mu) \operatorname{grad} T\right]=0$,

which reduces to the standard wave equation for the longitudinal wave, i.e. $\rho_{\mathrm{s}} \ddot{u}_{1}-(\lambda+2 \mu) \nabla^{2} \boldsymbol{u}_{1}=0$, only if the coupling term in grad $T$ is neglected, i.e. if the thermal expansion coefficient $\alpha_{t}$ is taken equal to zero. In the general case of thermoelasticity, Eq. ( 81$)$ is coupled to Eq. (80), and the solutions are a longitudinal quasielastic wave plus a quasi-thermal wave which are coupled. The quasi-elastic wave is damped and dispersed in a very complex manner. Its wavespeed is different from the pure longitudinal wave, i.e. $c_{1}=\left[(\lambda+2 \mu) / \rho_{\mathrm{s}}\right]^{1 / 2}$. There is a small corrective term $[51,58]$ that is given by

$$
V_{l}=c_{l} \sqrt{1+\epsilon}\left[1-\frac{\chi^{2} \epsilon(4-3 \epsilon)}{8(1+\epsilon)^{4}}+O\left(\chi^{4}\right)\right]
$$

with

$\epsilon=\frac{\left(3 K \alpha_{\mathrm{t}}\right)^{2} T_{0}}{\rho_{\mathrm{s}}(\lambda+2 \mu) C_{\mathrm{v}}}, K=\lambda+\frac{2}{3} \mu$, and $\chi=\omega \kappa /\left(\rho_{\mathrm{s}} C_{\mathrm{v}} c_{l}^{2}\right)$,

where $\omega$ is the angular frequency.

For most solids, at medium frequencies $(\sim$ a few $\mathrm{MHz}$ ), $\chi$ is very small (in the range of $10^{-5}-10^{-6}$ ), and as $\epsilon$, is also very small compared to $1, V_{l}$ can be simply approximated by $V_{l} \approx c_{l}(1+\epsilon / 2)$. In fact the correction is systematically very small, in the range of a few per cent. When dealing with anisotropic solids, the physics involved is much more intricate. In such a case, the three bulk modes are quasi-elastic waves which are coupled to the quasi-thermal wave. Nevertheless, the problem is not formally intractable and has been solved by various authors (see for instance the work done by Banerjee and Pao [59-61], or Sharma and Singh [62]). Starting with the general equations of thermoelasticity (including the influence of the relaxation tensor), e.g. Eqs. (71), (74), (76) and (77), these are rewritten after substitution of Eq. (74) into Eq. (71) in the form

$$
\begin{aligned}
& C_{i j k l} u_{l, k j}-m_{i j} T_{0} \Xi_{. j}=\rho_{\mathrm{s}} \ddot{u}_{i}, \\
& -q_{i, i}=\rho_{\mathrm{s}} C_{\mathrm{v}} T_{0} \doteq+T_{0} m_{i j} \dot{u}_{j, i},
\end{aligned}
$$

and

$\tau_{i j} \dot{q}_{j}+q_{j}=-k_{i j} T_{0} \Xi_{, j}$,

where

$\Xi=\left(T-T_{0}\right) / T_{0}, \quad m_{i j}=C_{i j k l} \alpha_{k l}$,

and

$\epsilon_{i j}=\left(u_{i, j}+u_{j, i}\right) / 2$.

The next step consists in considering plane harmonic waves for $u, q$ and $T$ of amplitudes $\geqslant, Q_{\mathrm{t}}$ and $\Theta$, and phase $\mathrm{j}(\omega t-k \boldsymbol{w} \cdot \boldsymbol{r})$. One then obtains the following system of homogeneous equations:

$$
\begin{aligned}
& \left(k^{2} C_{i j k l} w_{j} w_{k}-\rho_{\mathrm{s}} \omega^{2} \delta_{i l}\right) \|_{l}+\sqrt{-1} m_{i j} w_{j} k T_{0} \Theta=0, \\
& -\left(\omega \tau_{i j}+\sqrt{-1} \delta_{i j}\right) Q_{\iota j}+k \kappa_{i j} w_{j} T_{0} \Theta=0, \\
& -\sqrt{-1} k m_{i j} w_{i} T_{0} \omega \|_{j}+k w_{i} Q_{t i}-\rho_{\mathrm{s}} C_{\mathrm{v}} T_{0} \omega \Theta=0 .
\end{aligned}
$$

The secular equation associated to this set of homogeneous equations is a $7 \times 7$ determinant [ 60$]$ where the upper left part of it constitutes the $3 \times 3$ subdeterminant related to the uncoupled propagation of the bulk waves. the remaining part of the determinant being linked to 
the heat flux $\boldsymbol{Q}_{\mathrm{t}}$ and the temperature $\Theta$. The complete determinantal equation is eighth degree in $k$ with four pairs of complex roots corresponding to the four quasiwaves. and tenth degree in $\omega$ with two extra roots which are purely imaginary and thus do not contribute to propagation. It is possible to numerically compute the eigenvalues and the eigenvectors associated to such secular equation, and this has been done for various classes of symmetry where the determinantal equation specializes in much simplier forms [59.60], and thus to produce graphs for the slowness, velocity and wave surfaces. A pair of characteristic of such examples taken from these references are shown on Fig. 14. They clearly show the analogy between the thermal wave and the Biot mode.

\section{Inverse problems for the characterization of anisotropic porous media}

The objective is to determine some material parameters from accurate measurements done with ultrasonic techniques applied to the characterization of air-saturated porous media. We have seen in some of the previous sections that recovery of the anisotropic tortuosities was possible from wavespeed measurements (cf. Section 6), and that a fit of anisotropic viscous characteristic lengths was allowed from reflection coefficient experimental data (cf. Section 4). We can provide here further details on another inversion scheme. Let us start with Eq. (10) written in the following form:

$c(\omega)=\frac{c_{0}}{\sqrt{\alpha_{x}}}\left[1-\frac{\delta}{2}\left(\frac{1}{A}+\frac{\gamma-1}{B A^{\prime}}\right)\right]$.

This expression clearly shows that the slope of the straight line

$n_{\mathrm{r}}^{2}(\omega)=\left[\operatorname{Re}\left(\frac{c_{0}}{c(\omega)}\right)\right]^{2}=H\left(\frac{1}{\sqrt{(\omega)}}\right)$

is directly linked to the term

$(\hat{\imath}-1) \frac{1}{B A^{\prime}}+\frac{1}{A}$.

The next step is to perform a phase velocity measurement (a characteristic example is shown for an air-saturated plastic foam sample on Fig. 15) with the help of a phase spectrum algorithm (e.g. Refs. [27,28] in Section 6). By achieving such measurements in two gases having material parameters $(\gamma$ and $B$ ) sufficiently different (e.g. air and helium), the slopes are distinct. A simple linear regression is then providing the various parameters of interest. The tortuosity corresponds to $\omega \rightarrow+\infty$, i.e. $1 / \sqrt{\omega} \rightarrow 0$, and consequently is the intersection with the vertical axis of the straight line $n_{\mathrm{r}}^{2}(\omega)=$

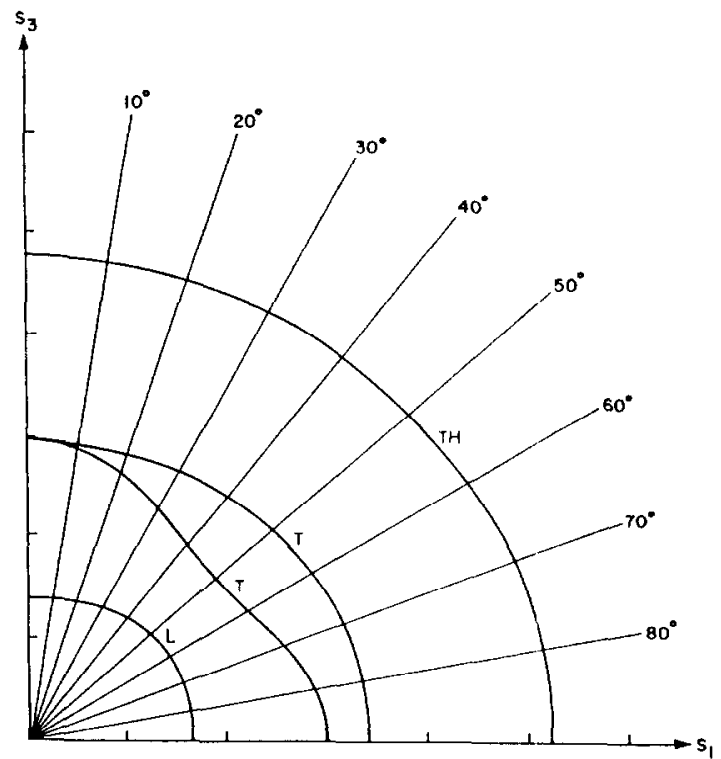

(a)

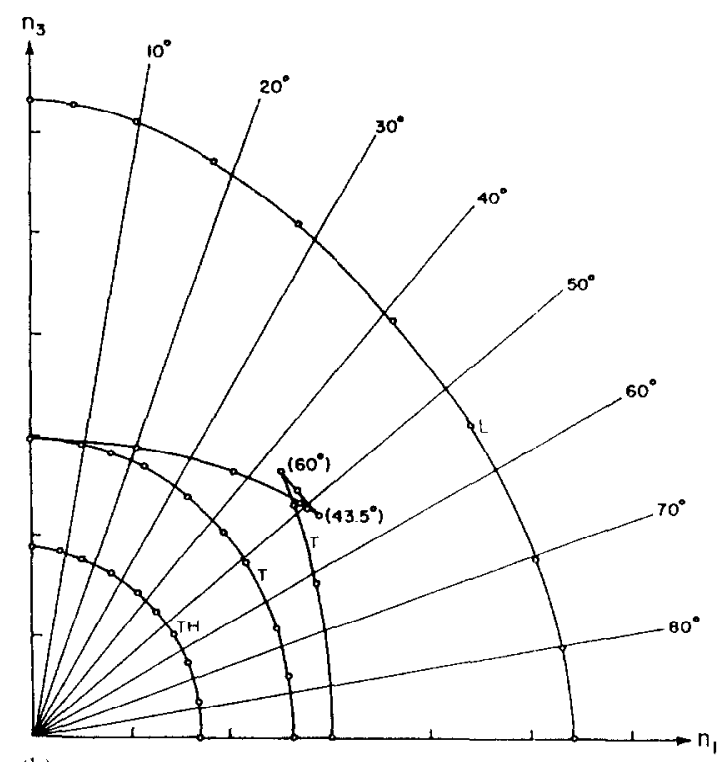

(b)

Fig. 14. Characteristic thermoelastic surfaces in anisotropic media. (a) Velocity surfaces in ${ }^{4} \mathrm{He}\langle 010\rangle$ at $0.85 \mathrm{~K}$ and $1 \mathrm{MHz}$. I unit $=$ $85 \mathrm{~m} \mathrm{~s}$. (b) Slownesses surfaces for sodium fluoride $\langle 010\rangle$ at $17.3 \mathrm{~K}$ and $3.3 \mathrm{MHz}, \quad 1$ unit $=0.104 \times 10^{5} \mathrm{~s} \mathrm{~cm}$. Adapted from Refs. $[59.60]$.

$H_{\text {linearized }}(1 / \sqrt{\omega})$. An example of such linear regression is shown on Fig. 16 for the same material (air-filled plastic foam). Then the values of the two slopes as obtained in the two gases enables to retrieve the two characteristic lengths $[63,64]$, see Fig. 17. It is interesting to note that the two lines should intersect at the origin. providing a cross-check on the precision of the determination of the tortuosity.

Another way to proceed is to design a nonlinear regression on the complete expressions for the phase velocity given in Section 2 [i.e. Eqs. (3) (7)], e.g. when 


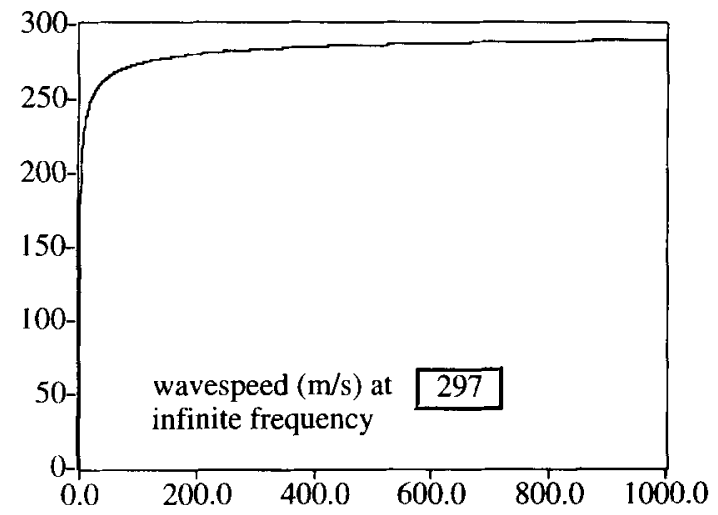

Fig. 15. Dispersion curve (wavespeed as a function of frequency) on an air saturated Tramico ${ }^{\Uparrow}$ plastic foam.

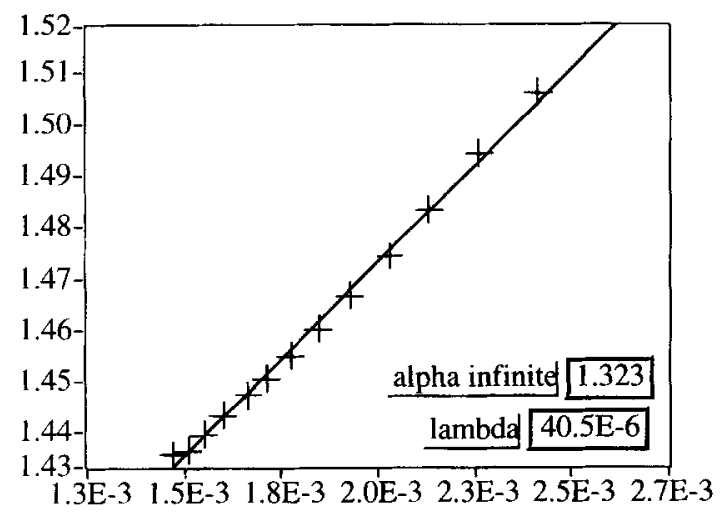

Fig. 16. Characteristic example illustrating the linear relationship which exists between the real part of the squared propagation index versus the inverse of the square root of frequency. The tortuosity is determined by linear regression as the intersection of the vertical axis. On this example using Tramico $\left.{ }^{(}\right)$and plastic foam, one obtains $\alpha_{\infty}=1.323$. The slope of the straight line yields $A=40.5 \mu \mathrm{m}$ for the viscous characteristic length, while $A^{\prime}=121.5 \mu \mathrm{m}$ as determined by the BET method $[8,9]$.

no high frequency approximation is done. In such case, this set of equations can be summarized in the following expression:

$\frac{\alpha_{\infty} c^{2}(\omega)}{c_{0}^{2}}=\Phi\left(\Lambda, A^{\prime}, \omega\right)$

where $\Phi\left(\Lambda, A^{\prime}\right)$ is expressed from Eys. (3)-(7). Eq. (91) in fact represents a set of $N$ equations, where $N$ is the number of wavespeed $c(\omega)$ measured as a function of frequency on the dispersion curve. This number $N$ is arbitrary large, and only depends on the sampling of the frequency domain (i.e. it is readily a few 10 s in size). Eq. (91) is then overdetermined as there are significantly more equations than unknowns (three in this case, i.e. the tortuosity and the two characteristic lengths). The following functional is then minimized in the leastsquare sense:

$\sum_{\omega}\left[\frac{\alpha_{\infty} c^{2}(\omega)}{c_{0}^{2}}-\Phi\left(\Lambda, \Lambda^{\prime}, \omega\right)\right]^{2}$ minimum

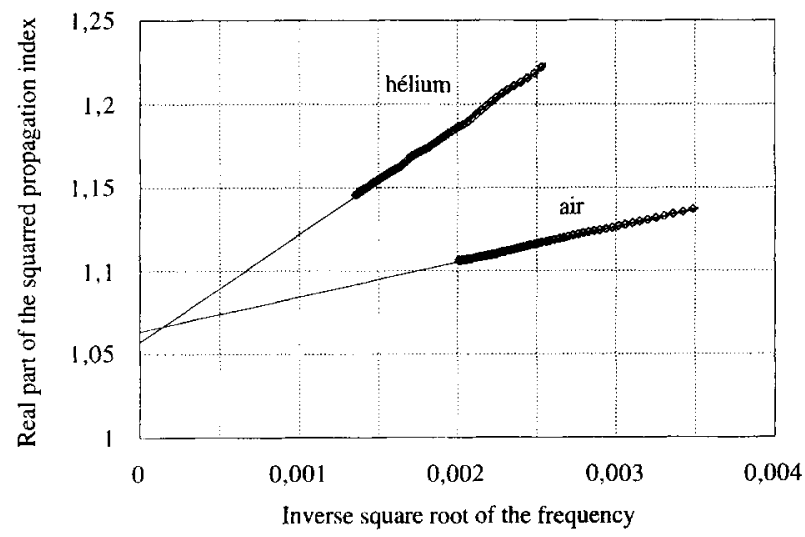

Fig. 17. Real part of the squared acoustical index of refraction for a plastic foam saturated by air or helium.

by using a Newton-Raphson algorithm [65], or any other appropriate numerical method (such as the conjugate gradient). For the Newton-Raphson method, one needs to solve the two following equations:

$\frac{\partial}{\partial \Lambda} \sum_{\omega}\left[\frac{\alpha_{\infty} c^{2}(\omega)}{c_{0}^{2}}-\Phi\left(A, \Lambda^{\prime}, \omega\right)\right]^{2}=0$
$\frac{\partial}{\partial \Lambda^{\prime}} \sum_{\omega}\left[\frac{\alpha_{\infty} c^{2}(\omega)}{c_{0}^{2}}-\Phi\left(\Lambda, \Lambda^{\prime}, \omega\right)\right]^{2}=0$.

The iterative solution is given by the following expressions which make use of the Hessian matrix associated to the functional:

$A^{(n+1)}=A^{(n)}-\left[\frac{\partial^{2}}{\partial A \partial A^{\prime}} \sum_{\omega}\{\}^{2}\right]^{(-1)} \frac{\partial}{\partial A^{\prime}} \sum_{\omega}\{\}^{2}$,

$A^{(n+1)}=A^{\prime(n)}-\left[\frac{\partial^{2}}{\partial A \partial \Lambda^{\prime}} \sum_{\omega}\{\}^{2}\right]^{(-1)} \frac{\partial}{\partial A} \sum_{\omega}\{\}^{2}$,

This method is completely general and should allow to determine the three material parameters from the complete dispersion curve. When anisotropy is present, the same process is applicable, and each dispersion curve as obtaincd along a given direction of propagation will evidently yield different values of the two anisotropic parameters (the thermal characteristic length being isotropic from its definition; e.g. see Ref. [6]).

\section{Concluding remarks}

Porous media are astonishing materials. Due to their diphasic structure at the same time being solid and fluid, they behave like an equivalent fluid, and sometimes as an elastic solid. In many cases the displacement fields in the two phases are coupled, and the full Biot theory must be used. When dealing with highly porous (i.e. $\phi$ 
close to 1) absorbing materials, the frame in most cases is not vibrating and the porous medium effectively acts as an 'equivalent fluid'. Most of the porous media are anisotropic. For instance, the anisotropy of porous rocks is related to their formation. Air-saturated porous media, such as synthetic plastic foams exhibit as well some anisotropy $[66,67]$, which is very often quite slight but still unmistakable. This anisotropy is related to the manufacturing process of the absorbing porous material (i.e. a polymerization chemical reaction for plastic foams). In the course of its fabrication, starting from a liquid mixture, the plastic foam expands by the proliferation of the bubbles, which create after their rupture the three-dimensional porous frame. During the expansion process, some very subtle physical phenomena occur which are responsible for gradients in the properties of the foam (e.g. the foam in the bottom of the tank becomes denser), for helerogeneousness and for anisotropy. One very intriguing finding that has been realized is linked to the orientation of the principal acoustical axes which do not coincide with the geometrical ones [18]. Again, this result is connected to the manufacturing of these materials. In the center of the tank, the principal axes are oriented along its sides, but when approaching the edges they become inclined because the expansion of the foam is restricted in that locations. Accordingly, the class of symmetry of this material must be close to the alleotropy which is found for wood species, that is the orientation of the principal axes being a function of the position. A further difficulty lies in the fact that these materials are also heterogeneous, as it is clearly visible from ultrasonic c-scan. Ultrasonic techniques are clearly valuable tools to decipher the intricacies of these anisotropic effects. We have shown in this review the type of theoretical models which are extensively used in that field, outlined the strong connection which exists with other coupled theories such as dynamical thermoelasticity (another coupled mechanism which is very similar is the coupling existing between the acoustic modes created in an air cavity and the solid vibrations of a cylindrical shell surrounding it). We have shown various approaches to tackle the anisotropic phenomena in acoustic materials by using ultrasonics, with a special mention for the inverse determination of constitutive parameters such as the tortuosity and the characteristic lengths (viscous and thermal). At present no other known method, besides ultrasound techniques, enables to provide detailed information on the anisotropy of these parameters including minute fluctuations and spatial variations. The state of the art in that field has been boosted during the last 5 years due to the many efforts in Le Mans and elsewhere [e.g. KU Leuven among the most active teams worldwide, having many fruitful collaborations with LAUM/IAM (Le Mans)].

\section{Nomenclature}

$A, C, F, M$, Biot's coefficients

$N, Q, R$

$B$

$c_{0}$

$C_{\mathrm{v}}$

e

E

$f$

j

$\boldsymbol{k}$

$K_{\mathbf{a}}$

$K_{\mathrm{e}}(\omega)$

$k_{0}$

$k_{0}^{\prime}$

$L$

$p$

Pr

$P_{0}$

$r$

$R_{\mathrm{i}}$

$R_{\mathrm{ti}}$

$m_{i j}$

$n_{\mathrm{r}}$

$s$

$T_{0}$

$T$

u

$U$

$U^{\prime}$

v

$w$

$Z_{\mathrm{f}}$

$Z_{\mathrm{m}}$

$Z_{\mathrm{n}}$

$\alpha(\omega)$

$\alpha_{\infty}$

$\alpha_{i}$

$\alpha_{t}$

$\tilde{\alpha}_{i j}(\omega)$

$\beta(\omega)$

$\gamma$

$\gamma_{i}$

$\delta$

$\epsilon_{k l}$

$\eta$

$\theta$

$\kappa$

$\lambda$

A

$A^{\prime}$

Young's modulus

frequency

imaginary unit

wavevector

sample thickness

acoustic pressure

Prandtl number

position vector cient

temperature

acoustic velocity

unit wave vector

fluid

tortuosity

refraction angle

direction

fluid viscosity

incidence angle

Lamé parameter square root of the Prandtl number

sound velocity in air

specific heat at constant volume

strain tensor of the fluid

compressibility modulus of air

equivalent compressibility modulus

static permeability

thermal permeability

atmospheric pressure

isotropic reflection coefficient

transversally isotropic reflection coeffi-

thermoelastic coupling constants

real part of the refraction index

stress in the saturating fluid

ambient temperature at rest

displacement vector of the frame

displacement vector of the saturating fluid

Displacement vector of the external fluid

characteristic impedance of the free fluid

characteristic impedance of the saturating

characteristic impedance of the saturating

fluid along normal direction

dynamic tortuosity

coefficient of linear expansion

tensor of dynamic tortuosity

relative dynamic compressibility

ratio of the specific heats

components of wave vectors along the $z$ -

viscous skin depth

strain tensor of the frame

isotropic thermal conductivity

viscous characteristic length

thermal characteristic length 
$\mu \quad$ Lamé parameter

$\mu_{\mathrm{s}} \quad$ structural damping coefficient of the frame

$v \quad$ Poisson ratio

$\rho_{\mathrm{s}} \quad$ mass density of the frame

$\rho_{\mathrm{f}} \quad$ mass density of the free fluid

$\sigma \quad$ component of the wavevector along $x$ axis

$\sigma_{i j} \quad$ components of the stress applied to the frame

$\phi \quad$ porosity

$\omega \quad$ angular frequency

\section{References}

[1] M.A. Biot, Acoustics, elasticity, and thermodynamics of porous media, twenty-one papers by M.A. Biot, edited by 1. Tolstoy, American Institute of Physics, 1992.

[2] P. Rebillard, Prédiction de l'impédance de surface des matériaux poreux stratifiés par la théorie de Biot, PhD Dissertation, Université du Maine, Mars, 1991.

[3] J.F. Allard, P. Herzog, D. Lafarge, M. Tamura, Appl. Acoust. 39 (1993) 3-21.

[4] P. Guignouard, L'absorption acoustique des matériaux poreux, PhD Dissertation, Université du Maine, Novembre, 1991.

[5] D. Lafarge, Propagation du son dans les matériaux poreux à structure rigide saturés par un gaz: definition de paramètres géométriques; analogies électromagnétiques; temps de relaxation et théorie universelle, PhD Dissertation, Université du Maine, September, 1993.

[6] J.F. Allard, Propagation of Sound in Porous Media: Modeling Sound Absorbing Materials, Chapman and Hall, London, 1993.

[7] D. Lafarge, J.F. Allard, B. Brouard, J. Acoust. Soc. Am. 93 (1993) 2474-2478.

[8] M. Henry, P. Lemarinier, J.F. Allard, J. Appl. Phys. 77 (1995) 17-20.

[9] P. Lemarinier, M. Henry, J.F. Allard, J.L. Bonardet, A. Gedeon, J. Acoust. Soc. Am. 97 (1995) 3478-3482.

[10] D.L. Johnson, J. Koplik, R. Dashen, J. Fluid. Mech. 176 (1987) 379-402.

[11] D.M.J. Smeulders, R.L.G.M. Eggels, M.E.H. van Dongen, J. Fluid. Mech. 245 (1992) 211-227.

[12] D.M.J. Smeulders, R.R. Van Hassel, M.E.H. van Dongen, J.K.M. Jansen, Int. J. Engng Sci. 32 (1994) 979-990.

[13] J.F. Allard, Y. Champoux, J. Acoust. Soc. Am. 91 (1992) 3346-3353.

[14] M. Melon, Caractérisation de matériaux poreux par ultrasons basse-fréquence $(20-500 \mathrm{kHz}), \mathrm{PhD}$ Dissertation, Université du Maine, October, 1996.

[15] B. Castagnede, M. Henry, P. I eclaire, L. Kelders, W. I auriks, C. R. Acad. Sci. Paris II 323 (1996) 177-183.

[16] B. Castagnede, N.R. Brown, M. Melon, C. R. Acad. Sci. Paris II 318 (1994) 1453-1457.

[17] P.B. Nagy, J. Acoust. Soc. Am. 99 (1996) 914-919.

[18] M. Melon, B. Castagnede, J. Acoust. Soc. Am. 98 (1995) 1228-1230.

[19] J.F. Allard, B. Castagnede, M. Henry, W. Lauriks, Rev. Sci. Instrum. 65 (1994) 754-755.

[20] M. Melon, D. Lafarge, B. Castagnede, N.R. Brown, J. Appl. Phys. 78 (1995) 4929-4933.

[21] C. Gazanhes, J.P. Herault, J. Phys. III 3 (1993) 2071-2086.
[22] D.L. Johnson, D.L. Hemmick, H. Kojima, J. Appl. Phys. 76 (1994) 104-114.

[23] D.L. Johnson, T.J. Plona, H. Kojima, J. Appl. Phys. 76 (1994) $115-125$.

[24] P. B. Nagy, I. Acoust. Soc. Am. 93 (1993) 3224-3234.

[25] P. Rasolofosaon, Propagation des ondes acoustiques dans les milieux poreux - Effets d'interface - (Théorie et expériences), $\mathrm{PhD}$ Dissertation, Université Paris 7, 1987.

[26] R.W.J.M. Sniekers, D.M.J. Smeulders, M.E.H. Van Dongen, H. Van der Kogel, J. Appl. Phys. 66 (1989) 45224524.

[27] W. Sachse, Y.H. Pao, J. Appl. Phys. 49 (1978) 4320-4327.

[28] N.R. Brown, B. Castagnede, W. Lauriks, M. Melon, C. R. Acad. Sci. Paris II 319 (1994) 393-399.

[29] B. Castagnede, J. Roux, B. Hosten, Ultrasonics 27 (1989) 280-287.

[30] B. Castagnede, J.T. Jenkins, W. Sachse, S. Baste, J. Appl. Phys. 67 (1990) 2753-2761.

[31] M.A. Biot, J. Acoust. Soc. Am. 28 (1956) 168-191.

[32] M.A. Biot, J. Appl. Phys. 27 (1956) 459-467.

[33] M.A. Biot, D.G. Willis, J. Appl. Mech. 24 (1957) 594-601.

[34] M.A. Biot, J. Appl. Phys. 33 (1962) 1482-1498.

[35] T.J. Plona, Appl. Phys. Lett. 36 (1980) 259-261.

[36] D.L. Johnson, Appl. Phys. Lett. 37 (1980) 1065-1067.

[37] D.L. Johnson, T.J. Plona, J. Acoust. Soc. Am. 72 (1982) 556-565.

[38] D.L. Johnson, T.J. Plona, C. Scala, Phys. Rev. Lett. 49 (1982) 1840-1844.

[39] M.E.H. Van Dongen, D.M. Smeulders, T. Kitamura, K. Takayama, Acustica 81 (1995) 63-70.

[40] M.D. Sharma, M.L. Gogna, J. Acoust. Soc. Am. 90 (1991) $1068-1073$.

[41] Auld, B.A., Acoustic Fields and Waves in Solids, vol. 1, 2nd edn, Wiley, Ncw York, 1990, pp. 221-236.

[42] B. Castagnede, C. Depollier, C. R. Acad. Sci. Paris II 317 (1993) 1007-1013.

[43] J.M. Carcione, J. Acoust. Soc. Am. 99 (1996) 2655-2666.

[44] A. Aknine, B. Castagnede, C. Depollier, C. R. Acad. Sci. Paris Il 324 (1997) 501-511.

[45] H. Deresiewicz, J.T. Rice, Bull. Seismol. Soc. Am. 53 (1963) 783-788.

[46] J.H. Rosenbaum, Geophysics 39 (1974) 14-32.

[47] K. Wu, X. Xue, L. Adler, J. Acoust. Soc. Am. 87 (1990) 2349-2358.

[48] B. Hosten, M. Deschamps, C. R. Acad. Sci. Paris II 299 (1984) $519-522$.

[49] B. Hosten, M. Deschamps, B.R. Tittmann, J. Acoust. Soc. Am. 82 (1987) 1763-1770.

[50] W. Nowacki, Thermoelasticity, Pergamon, Oxford, 1962.

[51] W. Nowacki, Dynamic Problems of Thermoelasticity, Polish Scientific, Warszawa, 1975.

[52] P. Chadwick, Thermoelasticity: The dynamical theory, in: Progress of Solid Mechanics, vol. 1, North Holland, Amsterdam, 1960, pp. 265-328.

[53] C. Cattaneo, C. R. Acad. Sci. Paris. 247 (1958) 431.

[54] G. Lebon, J. Lambermont, J. Mécanique 15 (1976) 579-594.

[55] D.D. Joseph, L. Preziosi, Rev. Mod. Phys. 61 (1989) 41-73.

[56] H.J. Maris, in: W.P. Mason, R.N. Thurston (Eds.), Physical Acoustics, vol. 8, Academic Press, New York, 1971 pp. 279-345.

[57] L. Landau, E. Lifchitz, Théorie de l'élasticité, Cours de physique théorique, vol. 7, Editions de Moscou, 1967, pp. 186-203.

[58] P. Chadwick, I.N. Sneddon, J. Mech. Phys. Solids 6 (1958) 223-230.

[59] D.K. Banerjee, Y.H. Pao, J. Acoust. Soc. Am. 56 (1974) $1444-1454$.

[60] D.K. Banerjee, Thermoelastic waves in anisotropic media, PhD. dissertation, Cornell University, NY, 1973.

[61] Y.H. Pao, D.K. Banerjee, J. Thermal Stresses 1 (1978) 99-112.

[62] J.N. Sharma, H. Singh, J. Acoust. Soc. Am. 85 (1989) 1407-1413. 
[63] P. Leclaire, L. Kelders, W. Lauriks, N.R. Brown, M. Melon, B. Castagnede, J. Appl. Phys. 80 (1996) 2009-2012.

[64] P. Leclaire, L. Kelders, W. Lauriks, C. Glorieux, J. Thoen, J. Acoust. Soc. Am. 99 (1996) 1944-1948.

[65] W.H. Press. B.P. Flannery, S.A. Teukolsky, W.T. Wetterling,
Numerical recipes. The Art of Numerical Computing, Cambridge UP, New York, 1986.

[66] L.J. Gibson, M.F. Ashby, Cellular Solids, Structure and Properties, Pergamon, Oxford, 1988.

[67] A.T. Huber, L.J. Gibson, J. Mater. Sci. 23 (1988) 3031-3040. 